(Aus dem physiologischen Institute zu Halle a. d. S.)

\title{
Experimenteller Beitrag zur Physiologie des Ohrlabyrinthes.
}

\author{
Von \\ Dr. med. F. Matte. \\ Assistent am physiologischen Institut zu Halle a. d. S.
}

Hierzu Tafel VI und eine Textfigur.

\section{\$1. Historisches.}

Bevor wir in die Darstellung der eigenen experimentellen Untersuchungen eintreten, sollen noch einmal die von Flourens, dem Entdecker der eigenthümlichen Erscheinungen nach Labyrintheingriffen, angestellten Versuche und deren Resultate mit kurzen Worten erwähnt werden. Dies erscheint um so nothwendiger, als es nach allen den in zahlreichen Schriften enthaltenen spärlichen, oft nur wenige Sätze umfassenden Notizen den Anschein hat, als ob diese grundlegenden Untersuehungen der Vergessenheit anheimfallen. Vor Allen finden sich in Flourens' Recherches expérimentales ${ }^{1}$ ) mehrere Capitel, deren Inhalte ein grösseres Interesse gebührt.

Zunächst seien die Recherches sur les conditions fondamentales de l'audition ${ }^{2}$ ) erwähnt. Es handelt sich dabei um Versuche mit Entfernung der Trommelfelle, Extraction der Gebörknöchelchen (Columellen), Zerstörung der fenestra rotunda et ovalis und endlich Eingriffe auf die periphere Endausbreitung des Hörnerven selbst. Bei allen auf ihre Hörfähigkeit nach den Eingriffen zu prüfenden Thieren wird zunächst durch einen Schirm jede directe Einwirkung der Luftbewegung, welche auch gefühlt werden kann, ausgeschlossen, ferner jede Fortpflanzung von Erschütterungen

1) Recherches expérimentales sur les propriétés et les fonctions du système nerveux dans les animaux vertébrés. Paris 1842.

2) 1. c. $438 \mathrm{ff}$. 
sorgfältigst vermieden und endlich, um keinerlei Täuschungen durch Gesichtswahrnehmungen zu unterliegen und gleichzeitig, „um die Aufmerksamkeit der Thiere auf den zu prüfenden Sinn zu concentriren", die Augen verschlossen.

Das bekannte Resultat dieser Untersuchungen ist: der Hauptantheil beim Hören kommt der Schneckenausbreitung des Hörnerven $九 1$, welche sogar absolut nothwendig ist. Eine doppelseitige Zerstörung dieses Antheiles des Labyrinthes bedingt demnach vollkommene Taubheit ${ }^{1}$ ) (p. 451). Alle übrigen Bestandtheile des Gehörorganes haben im Verhältniss zur Schnecke nur geringe Bedeutung.

Eine sehr wichtige, eigenarte Function kommt nun aber dem Bogengangapparate des Labyrinthes zu, denn dessen Verletzung bedingt zunächst die unmittelbar nach der Verletzung auftretende Schmerzhaftigkeit, welche mit der Verheilung der Wunde abnimmt, ferner ist sie von einer stürmischen Kopfbewegung gefolgt. Es besteht eine ïberraschende $\Delta$ bhängigkeit der Richtung der Kopfund Körperbewegungen von der Lage der einzelnen Bogengänge bei Vögeln und bei Säugethieren. Dem zu Folge treten nach Verletzung der canales horizontales eine horizontale Kopfbewegung und Umdrehungen der Thiere um sich selbst auf, nach Verletzung der canales verticales anteriores: verticale Kopfbewegungen und Ueberschläge nach vorn, nach Verletzung der canales verticales posteriores: auch verticale Kopfbewegungen, aber Ueberschläge rückwärts. Bei Säugethieren sind die Bewegungsstörungen zwar von geringerer Heftigkeit, dafiir aber anscheinend von grösserer Constanz. In der Rube treten die Störungen zurück, ihre Intensität erwacht und wächst bei Bewegungen. Ueber das sonstige Verhalten der Thiere erfahren wir nur wenig. Oft hat die Beobachtungszeit über ein halbes Jahr gewährt, die Bewegungsstörungen baben nahezu von derselben Intensität und demselben Charakter bestanden. Im Uebrigen verhalten sich die Thiere normal und sind bei genügender Sorgfalt betreffs ihrer Ernährung geradezu gemästet gewesen.

1) Fine isolirte Schneckenzerstörung bei Vögeln ist Flou rens nicht gelungen, es ist gleichzeitig immer das Vestibulum betheiligt gewesen. Dagegen ist diese Operation bei Kaninchen geglückt, wobei Folgendes festgestellt wird: l'ouie a été détruite, et les mouvements singuliers qui suivent la section des canaux semi-circulaires n'ont point paru! (p. 492, Anm. 2.) 
F l o uren s' Resultat bezüglich der Function des N. acusticus ist demnach folgendes ${ }^{1}$ ): le nerf acoustique n'est pas un nerf simple; c'est un nerf complexe et qui se compose de deux nerfs très distincts: le nerf du limaçon et le nerf des cananx semi-circulaires.

Le premier de ces nerfs le nerf du limaçon, est le vrai nerf auditif le limaçon est le vrai siège du sens de l'ouĩe.

L'autre nerf, le nerfdes canax semi-circulaires, n'est pas un nerf des sens: la section des canaux semicirculaires ne détruit pas l'ouie; elle la rend même plus vive, puisqu'elle la rend douloureuse. Le nerf des canaux semi-cireulaires est un nerf spécial et propre. Il est d oué de la facultésingulièred'agirsurla direction des mouvements.

Aus diesen Worten geht die Vorstelling, welche Flourens von der Function dieses Hörnervenantheiles hat, klar hervor. Er fasst denselben nicht als eigentlichen Sinnesnerven auf und vermeidet auch eine Theorie aufzustellen.

Das Hauptverdienst von $\mathrm{Goltz}^{2}$ ) besteht nun darin, dass er gerade die Bedeutung der peripheren Organe erkannt bat, welche nach ihm als e in e A r vo n Sin nesorga $\mathrm{n}$ aufzufassen sind. Dem mit ihnen verbundenen Nervenfaserantheile des $N$. actsticus kommt sonach die spezifische Energie zu den Centralorganen, die Empfindung der Kopfhaltung zu vermitteln.

In meiner 1892 erschienenen Dissertation $^{3}$ ) ist die Litteratur der Ohrphysiologie, welche sich mit der Funktion der Bogengänge beschäftigt, soweit sie zugänglich und von Interesse gewesen ist, berïcksichtigt. Ueber die seither veröffentlichten Arbeiten finden sich Referate von $\mathrm{Hensen}{ }^{4}$ ) und von Kreidl ${ }^{5}$ ) vor, es scheint demnach überflüssig, hier auf deren Inbalt zurückzukommen; theilweise findet sich dazu Gelegenheit bei der folgenden Darstellung.

1) 1. c. p. 492.

2) Pflüger's Archiv. III. p. 172.

3) Ein Beitrag zur Function der Bogengånge des Labyrinths. Diss. inaug. Halle 1892.

4) Vortrag gegen den sechsten Sinn. Arch. f. Ohrenheilk. XXXV. Bd.

5) Weitere Beiträge zur Physiologie d. Ohrlabyrinthes. Versuche an Krebsen. Sitzungsher. d. kais. Acad. d. Wiss. i. Wien. Mathem. naturw. Cl. Bd. CII. Abth. III. 1893. 


\section{§ 2. Experimentelles.}

Die ersten Versuche, welche in der Dissertation veröffentlicht sind, beziehen sich auf Sondirungen einzelner und mehrerer Bogengänge. In mehreren Zeitschriften sind darüber mehr oder weniger umfangreiche Referate ersehienen. Trotzdem soll hier noch einmal kurz auf die Methode und die Versuchsresultate der Sondirungen zurückgekommen werden. Der in der Dissertation zunächst angenommene Vorschlag Spamers, die Nomenclatur betreffend, ist aufgegeben und werden die einzelnen Bogengänge bezeichnet, wie zumeist iiblich, mit

canalis horizontalis s. externus (H. C.),

canalis verticalis anterior s. anterior (Längsverticalcanal

L. V. C.),

canalis verticalis posterior s. posterior (Querverticalcanal (Q. V. C.).

Bevor wir nun zur Darstellung der Versuche übergehen, sollen einige Worte über Instrumentarium und Operationsverfahren vorausgeschickt werden, dies geschieht um so mehr, als die überreiche Mannigfaltigkeit und Complicirtheit der von Ewald ${ }^{1}$ ) für Labyrinthoperationen angegebenen Instrumente manchen vor experimentellen Prüfungen direct abschrecken möchten.

In Allgemeinen brauche ich ein Scalpell, ein tenotomartiges Messerchen, einige feine Mikroskopirpincetten, feine Hakenpincetten mit gebogenen Branchen (Irispincetten), feine spitze Stahlnadeln, feine Scheeren, Nadeln ete. Für einzelne Operationen ausserdem Galvanocauter oder Platindraht, schwarze Rosshaarsonden nnd ganz feine Bäkchen, die für bestimmte Zwecke aus feinen Karlsbader Nadeln gebogen und an einem mit einer Stellschranbe versehenen Holzgriffe befestigt werden. Sämmtliche Instrumente und Sonden werden zuror gut desinficirt, wie iberhaupt die Anforderungen der modernen Wundbehandlung die möglichste Berïcksichtigung finden.

Die Thiere werden in ein reines Handtuch gewiekelt, so dass keinerlei willkürliche Bewegungen des Körpers oder der Extremitäten gemacht werden können. Vor der Operation werden die

1) Physiologische Untersuchungen über das Endorgan des N. octavus. Wiesbaden 1892. p. 53-74 etc. 
Federn am Hinterkopfe möglichst kurz abgeschnitten und die Hant gereinigt und desinficirt. Nun beginnt die Einleitung der Narcose mit Chloroforn-Jether aa ${ }^{1}$ ). Der Hautschnitt wird mit dem Scalpell in der Mittellinie über den Hinterkopf und Nacken gleichweit nach oben und unten vom Ansatz der Nackenmuskeln an der $\mathrm{Li}$ nea occipit. ext. angelegt von einer Länge, dass durch Verschiebung der Haut das Operationsterrain bequem freigehalten werden kann. Die geringe Hautblutung ist leicht gestillt. Darauf folgt die Trennung der beiden Nackenmuskeln in dem von Cyon ${ }^{2}$ ) angegebenen weissen Streifen mit dem tenotomartigen Messerehen sofort bis auf den Knochen und die seitliche Ablösung der Muskelinsertionen, bcsonders der vor der Trennungslinie gelegenen Partien, natürlich auch mit möglichster Schonung, um nicht zu bedeutenden Ausfall von Muskelactionen herbeizuführen ${ }^{3}$ ). Die Blutung der kleinen Wundfäche ist meist sehr geringfügig. Liegt nun die knöcherne Ohrkapsel, durch deren dünne Wandung besonders das Bogengangskreuz durchschimmert, sauber vor uns die seitlichen Muskelpartien werden mit dem Daumen und Zeigefinger der linken Hand zurückgebalten, während der Kopf des Thieres in der linken Hollhand ruht - so erfolgt die Abtragung der das Geliörorgan deckenden äusseren Knochenlamelle, wobei bei guter Tagesbeleuchtung mit directem oder reflectirtem Licht ohne jede weitere Blutung eine saubere Präparation der Bogengünge gelingt. Es werden dazu feine Mikroskopirpincetten oder mit besonderem Vortheile die erwähnten Hakenpincetten mit gebogenen Branchen verwendet. Assistenz habe ich nicht gebraucht, die Finger der linken Hand halten den Kopf der Taube nach jeweiligem Bedarf. Dabei ist jedoch darauf zu achten, dass nicht die Luftzufubr durch Compression der Nasenöffnungen vermindert wird.

1) Bezüglich des Erbrechens gehen meine Erfahrungen dahin, dass in der Mehrzahl der Fälle die angewendeten Narcotica verantwortich zu machen sind, da auch normale Thiere erbrochen haben, die zur Prüfung narcotisirt worden sind.

2) Methodik der physiologischen Experimente und Vivisectionen. Giessen 1876 .

3) Uebrigens erscheinen bei dem angegebenen Operationsverfahren derartige Besorgnisse nicht gerechtfertigt. Es zeigen sich nämlich bei Thieren, an denen nur die vorbereitenden Operationen angestellt sind, niemals augenfällige Bewegungsstörungen. 
Ueber die Vortheile eines Ewald'schen Taubenhalters babe ich keine Erfahrungen, ich bedarf keines besonderen Apparates. Die meisten Operationen sind mit unbewaffnetem Auge unter obigen Bedingungen ausgeführt, nur hin und wieder dient eine Lupe zur Orientirung. Bei den zur Darstellung der Folgeerscheinungen referirten Fällen ist keinerlei Nebenverletzung gemacht worden. Nach beendigtem Eingriffe wird das Operationsterrain sorgfältig mit reiner Watte gesäubert, etwaige Flüssigkeit aufgesogen, endlich die Hautwunde mit einigen Nähten geschlossen. Die Heilung geschieht binnen kurzer Zeit.

\section{a) Sondirungen.}

Nach vorsichtiger Freilegung der zu sondirenden Canäle geschieht ihre Eröffnung mit einer feinen Stahlnadel, die Erweiterung der punktförmigen Oeffnung mit einer Staarnadel. Die Beobachtungen währen bei den einzelnen Versuchen verschieden lang. Bei jeder Versuchsanordnung wird jedoch an je einem Versuchsthiere nach 24 Stunden Section gemacht, um die Lage und Länge der Sonden festzustellen.

x) Sondirungen der beiden canales horizontales.

Die Eröffnung der freipräparirten Canäle geschieht wenige mm vor der Kreuzung mit den canales vertic. post. Die Sonden werden sowohl in der Richtung nach der Ampulle hin als auch nach der entgegengesetzten Richtung soweit vorgeschoben, bis dieselben auf Widerstand stossen. Sogleich tritt lebhaftes Pendeln in der horizontalen Ebene auf, an das sich bei Bewegungsversuchen eine Neigung nach rechts oder links umzudrehen und nach vorne überzustiirzen anschliesst.

Bei mehrwöchentlicher Beobachtung; wobei die Sonden 3 Tage lang liegen geblieben sind, bestehen die erwähnten Störangen zunächst in gleicher Intensität. Dazu gesellt sich sehr bald eine vollständige Verdrehung des Kopfes in der Medianebene: das Hinterhaupt rubt mit beträchtlichem Drucke auf dem Boden, der Schnabel mitten auf der Brust. Jede Anregung wird mit einer unverkennbaren Aeusserung des Unwillens beantwortet. Zu Bewe. gungen angetrieben geht das Thier rückwärts, indem es in der abnormen Stellung verharrt. Die Sondenentfernung bringt zunächst keine Aenderung der Erscheinungen: der Kopf wird wiederum mit 
dem Hinterhaupte fest auf den Boden gestiutzt, der Schnabel ist nach oben gerichtet. Im Käfig werden Schwanz und hintere Extremitäten aufrecht gegen das Gitter gestemmt, die Längsaxe des Körpers bildet mit dem Boden einen Winkel von etwa $70^{\circ}$. Bereits am Nachmittag hat das Thier die normale Kopf- und Körperhaltung eingenommen. Kopfpendeln und Vorwärtsbewegung in Schlangenlinien sind noch deutlich. In den folgenden Tagen nehmen auch diese Erscheinungen mehr und mehr ab, es bleibt zuletzt nur noch die Neigung leicht im Bogen zu laufen.

Eine 14 Tage später an demselben Thiere vorgenommene Sondirung, welche aber nur links gelingt, ruft das eben gezeichnete Bild wieder hervor. Auch hier schwinden die Störungen bald, es bleibt bis zur Section nur die Neigung bei Bewegungen Umdrehangen nach links auszuführen.

\section{B) Sondirungen der beiden canales verticales anteriores.}

Um diese Art Sondirung auszuführen, bedarf es gar keines Eingriffes auf die Muskulatur. Unmittelbar über der Insertionsstelle wird über beiden Nackenmuskeln die äussere Knochenlamelle anfgebrochen, das pneumatische Knochengewebe entfernt und allmählich bis auf die Canäle vorgedrungen, wobei der über dem Canal gelegene Sinus deutlich die Richtung kennzeichnet. Man befindet sich anf diese Weise oberhalb des durch die Blutgefässe gefährdeten Terrains und operirt ohne jede Blutung noch Muskelverletzung. Die Oeffnungsstellen liegen $3 \mathrm{~mm}$ von den Ampullen entfernt, die Sonden werden in der Canalrichtung vorgeschoben. Die Pendelbewegungen treten auf beiden Seiten praecis ein, ihre Intensität ist nach doppelseitiger Sondirung bedeutender, in ihrer Gesammterscheinung - von oben gesehen - haben dieselben eine auffallende Aehnlichkeit mit einer stehenden 8. Allmählich ändern sich beide Pendelrichtungen, indem der Kopf nunmehr gewissermassen in der Resultante beider Richtungen, d. h. in der Medianebene heftig aufund abbewegt wird. Diese Aenderung tritt, wie gesagt, allmählich ein; es kommen aber noch Bewegungen in beiden Canalrichtungen praecis zum Vorsehein. Wird das Thier auf den Boden gesetzt, so tritt bei willkürlichen Körperbewegungen sofort ein Niederstürzen nach vorn ỉber ein, das Thier kann sich zunächst nicht in die normale Haltung zurüekbringen. Es schiebt mit der Stirn auf dem Boden vorwärts und zeigt dabei eine Neigung, leichte Drehungen 
nach rechts oder links zu vollfiihren. In die normale Haltung zurückgebracht, wird dieselbe nur mit Mühe durch Flügelausbreiten, Beinspreitzen und Aufstiitzen auf den fächerartig entfalteten Schwanz erhalten. Gegen die durch die Canalrichtung bestimmten Kiopfbewegungen findet das Thier auf diese Weise Schutz. D i e Pen delbewegungen in der Medianebene bewirken dagegen, da sie gewissermassen eine $\mathrm{S} u \mathrm{mmation}$ und $\mathrm{Com}$ bination der beiderseitigen Reizungen vorstellen, stets das beschriebene Vornüberstürzen.

Die Section am nächsten Tage ergibt eine vollkommene Sondirung der Canäle bis zu den Vereinigungsstellen mit den Horizontalcanälen.

Bei längerer Beobachtung derartiger Sondirungen ist das Verhalten der Thiere analog wie oben: starkes Pendeln in den Ebenen des Ganges, Umdrehungen, Niederstïrzen. Nach Entfernung der Sonden tritt Besserung ein. Es bleiben eine auffallende wochenlange Ungeschicklichkeit der Thiere beim Fressen, allgemeine Unsicherheit der Kopf- und der Körperbewegungen.

1) Sondirungen der beiden canales verticales posteriores.

Die Eröffnung der Canäle wird genau an der Kreuzungsstelle derselben mit den canales horizontales vorgenommen. Die Sonden werden beiderseits nach den Ampullen hin und von den Ampullon weg eingeführt. Bei der Sondirung auf der linken Seite nach unten, d. h. nach der Ampulle hin, zeigt das Thier sehr heftige Reactionserscheinungen. Die sogleich auftretenden lebhaften Pendelbewegungen in der Ebene dieses Ganges steigern sich bis zur Schraubenverdrehung des Kopfes nach links und oben um eine auf der Bogengangsebene senkrecht stehend gedachte Linie als Achse. Nachdem sich das Thier wieder beruhigt hat, wird durch die nachfolgende Sondirung rechts wieder eine bedeutende Steigerung der Erseheinungen hervorgerufen. Die Pendelbewegungen erfolgen nun bald in der Ebene des linken, bald in der des rechten Bogenganges, sie gewähren in ihrer Gesammtheit den Eindruck, als wenn sie in Form einer liegenden $\infty$ vor sich gingen. Bei Gehversuchen zeigt sich eine Neigung nach hinten über zu stürzen, wogegen das Thier anscheinend mit grosser Anstrengung (Fligelausbreiten, Beinspreitzen, Entrollen der Schwanzfedern) ankämpft. Mit allen zur Verfügung: 
stehenden Hülfsmitteln wird das Ueberschlagen rüickwärts zwar vermieden, dagegen kommt es dabei zum Rückwärtsgehen.

Die längere Beobachtung solcher Thiere bestätigt auch bei diesen Versuchsanordnungen eine allmähliche Besserung nach Entfernung der Sonden. Zunächst werden nur noch Umdrehungen nach rechts und links and Pendeln beobachtet. Auch die Pendelbewegungen verschwinden, aus den Umdrehungen wird Bogenlaufen, bis nach etwa 10 Tagen so ziemlich alle Erscheinungen verschwunden sind.

Zu erwähnen bleibt noch, dass bei einem derartigen Versuche trotz bedeutender Schwierigkeiten eine Wiederholung der ganzen Operation gelungen ist. Der Erfolg ist unzweifelhaft. Es treten von neuem Pendelbewegungen in den verletzten Ebenen besonders bei Bewegungen auf, daneben Umdrehungen.

d) Sondirnngen eines canalis vert. ant. einerseits und eines canalis vert. post. andererseits und umgekelurt.

Das Resultat dieser Versuchsanordnungen lässt sich dahin zusammenfassen, das eine deutliche Verstärkung der Kopfbewegungen eintritt, was sich aus der nahezu parallelen Lage der sondirten Theile der Bogengänge erklären lässt. $\mathrm{Zn}$ diesen Störungen gesellen sich bei spontanen Bewegungen eine grosse Unsicherheit des Körpers, die sich bis zum Ueberschlagen steigert. -

Da wir uns die Wirkung der Sonden kaum anders als isolirte mechanische Reizung der Nervenendapparate in den betreffenden Ampullen vorstellen können, so tragen auch die unmittelbar nach der Sondirung auftretenden Erscheinungen, die in der Hauptsache in Pendelbewegungen des Kopfes in der Ebene des sondirten Canales bestehen, deutlich den Charakter von Reizerscheinungen. Dafuir spricht ausserdem die rasehe Abnahme der Erscheinungen wach Entfernung der Sonden und das Wiedererwachen bereits verschwundener Störungen nach Wiedereinführung derselben.

\section{b) Exstirpationen.}

Um eine vollkommene Exstirpation zu erleichtern, werden die canales horizontales freigelegt. Die begleitenden Sinus werden mit einem glïhenden Platindraht, der ans besonderen Riicksichten statt des Galvanocauters benutzt wird, zwischen Ampullen und Bogengangkrenz in einer Ausdehnung von einigen mm verödet. Die Verödung muss reeht vorsichtig und vollkommen sein, damit nicht 
etwa nachträglich durch Knochensplitterungen eine unangenehme Blutung das winzige Operationsterrain überschwemmt. Die Verödung ist kenntlich an der Verfärbung und an dem Abbröckeln der verkohlten Partien. Dann werden die Canäle mit der Scheere durchschnitten. Ist bisher alles gut gelungen, so dringt man ohne Gefahren durch die Knochenbälkchen in die Tiefe auf die Ampullen vor und bobrt sie mit einer spitzen Stahlnadel an. Aus den kleinen Oeffnungen dringt lymphatische Flüssigkeit vor, die mit feinen um eine spitze Pincette gewickelten reinen Wattetupfern aufgesogen wird. Die Oeffnungen werden nach Bedürfniss erweitert und der Inhalt herausgezogen. Hierzu werden entweder feine Hakenpincetten mit gebogenen Branchen benutzt oder, was ich besonders empfehlen kann, äusserst feine aus Karlsbadernadeln zu diesem Zwecke gebogene Häkchen, mit denen sowohl sondirt wie etwaiger Inhalt bequem herausbefördert werden kann. Folgt der Inhalt nicht sofort, so braucht man die Häkchen in den Oeffnungen nur einige Male umzudrehen, es wird dann sicher der Inhalt gefasst und kann lerausgezogen werden. Die übrige Labyrinthwand kann man dann noch vollkommen losbrechen, um sich davon zu überzeugen, dass das Gewiinschte entfernt ist. Die hervorgezogenen Gewebstheile kommen sofort in die bereitstehenden Flüssigkeiten zur mikroskopischen Untersuchung.

\section{a) Einseitige Totalexstirpationen.}

$\mathrm{Zu}$ obigen für die Exstirpationen von Labyrinththeilen im allgemeinen geltenden Bemerkungen ist noch hinzuzuftigen, dass es mir mehrfach geglückt ist, obne vorhergehende Verödung der Sinus im vorderen oberen Bogengangkreuzquadranten bis auf die knöchernen Ampullen des canalis horizontalis und canalis vert. ant. vorzudrịngen, dieselben anzubohren, die Bohröffnungen gentigend zu erweitern und den Inbalt lierauszuziehen, dann von derselben Oeffnung aus nicht nur den Inhalt der pars inferior, sondern auch die Ampulle und den häutigen canalis vertic. post. herauszubeförden. Dazu dient mit besonderem Vortheile ein oben beschriebenes feines Häkchen, das etwa 1-2 mm vor dem gekriimmten Ende stumpfwinklig abgebogen ist. Ueberbaupt gelingt es mit einem solchen Instrument das ganze Labyrinth ebenso schonend wie grüdlich abzusuchen.

Was nun das Verhalten derartig operirter Thiere anbetrifft, 
so stimme ich mit Ewald's Beobachtungen vollkommen überein. Unmittelbar nach der Operation treten Gleichgewichtsstörungen (Kopf- und Körperbewegungen) nach der operirten Seite hin anf. Ihre Dauer ist kurz und individuell verschieden. Nach dieser Zeit bessert sich das Verhalten schnell. Die Tauben können gehen, laufen und wohl auch fliegen, sie nehmen stets selbständig Nahrung zu sich. Noch vor Ablauf etwa einer Woche tritt allmählich in der Ruhe eine schiefe Kopfhaltung nach der operirten Seite hin auf und nan gelingt es durch Erschrecken, nach heftigen Bewegungen (Flug- und Fluchtversuchen) eine vollkommene Verdrehung des Kopfes (schraubenförmig nach der operirten Seite) hervorzurufen, sodass das Hinterhaupt mit dem Scheitel den Boden beriuhrt und der Schnabel fast senkrecht nach oben steht. Zuweilen ist es auch gelungen, bei einem Thiere, das in der Ruhe noch keinerlei Verdrehungen gezeigt hat, durch heftiges Erschrecken eine to t a le Kopfverdrehung (bis zum Ew ald 'schen Eudstadium) herbeizuführen. Es scheinen in solchen Fällen diese merkwürdigen Störungen gewissermassen latent gewesen zu sein. Von der Zeit an wird der Kopf kaum noch jemals normal gebalten, mindestens ist eine schiefe Kopthaltung, meist sogar eine Drehung um nahezu $90^{\circ}$, sodass das Auge der operirten Seite nach unten, das der gesunden Seite nach oben sieht, zu constatiren.

Bei Bewegungsversuchen kommt es dann zu Umdrehungen. Fliegenkönnendie Tauben vonder Zeitan nicht $\mathrm{mehr}$. Sie machen zwar noch Versnche dazu. Es lässt sich dabei beobachten, dass einzelne Thiere in der Luft Umdrebungen um die Längsaxe des Körpers nach der operirten Seite hin machen, um dann wie eine todte Masse in dumpfem Falle niederzustiirzen und diese Umdrehungen noch fortzusetzen. Das ganze Verbalten erinnert dann lebhaft an die Rollbewegungen eines quer durch den Kopf galvanisirten Kaninchens. Auch kommt es vor, dass einzelne Thiere bei Flugversuchen riickwärts sich bewegen, ohne sich recht vom Boden erheben zu können. Auf alle derartigen Bewegungsversuche tritt dann stets eine hochgradige Kopfverdrehung von längerer Dauer ein.

Der Umstand nun, dass diese eigenthümlichen Kopfverdrehungen erst nach einigen Tagen (in max. 6-8 Tagen, in welcher Zeit die Thiere ges c hont werden), dann nach heftigen Bewegungen oder in Angstzuständen eintreten, endlich die Thatsache, dass sie 
nach Eingriffen auf den Bogengangapparat der anderen Seite dauernd verschwinden, macht es wahrscheinlich, dass es sich hier nm Wirkungen handele, welche naturgemäss von der gesunden Seite ausgehen müssen, weil die operirte Seite ohne nervöse Elemente, also auch ohne Erregungen bleibt. Fïr die ersten Tage nach der Operation und für die Ruhe genügen die übrigen noch vorhandenen Vorrichtungen zur Erhaltung des Kopfgleichgewichts, bei heftigen Bewegungen, welche noch dazu unter Umständen ganz plötzlich coordinirt werden sollen, wirkt die von der gesunden Seite ausgehende Erregung der Art, dass es in Folge des Ausfalles auf der operirten Seite zu abnormen Wirkungen kommt.

Eine ausreichende Erklärung für dieses merkwürdige Verhalten zu gebeu, ist sehr schwer. Um dennoch zu einem Verständniss zu gelangen, könnte man die Wirkungen bei galvanischer Durchströmung des Kopfes am Menschen von einem Ohre zum andern zu einem Vergleiche heranziehen. Es ist nämlich eine bekannte Thatsache, dass die Versuchsperson auf einem Stuhle sitzend bei der Querdurchleitung entsprechend starker Ströme an der Stelle, wo der Strom austritt, also an der Kathode, die Empfindung hat, als müsste sie vom Stuhle nach dieser Seite berabsinken, und gegen diese Empfindung wehrt sie sich durch eine entsprechende Neigung des Kopfes und Körpers nach der anderen Seite, also nach der Anode. Und letztere Bewegung tritt thatsächlich in $\mathrm{Er}$ scheinung.

Hier möchte ich dann gleich noch auf das bereits von Ewald beobachtete Verhalten einseitig labyrinthloser Thiere bei galvanischer Querdurchströmung aufmerksam machen. Es hören nämlich, wenn sich die Anode auf der gesunden Seite befindet, die Kopfverdrehungen auf. Die plausibelste Erklärung hierfür ist wohl die Annahme einer Anelectrotonusentwicklung auf der gesunden Seite, welche eine Hemmung der von hier ansgehenden Erregungen bedingt (Labyrinth-(Ausfalls)-Reaction E wald's) ${ }^{1}$ ). Bei hinreichender Stromstärke werden die von hier ausgehenden Wirkungen ganz beseitigt und während der Stromesdauer gewisser. massen eine doppelseitig labyrinthlose Taube hergestellt, indem der Ausfall auf der operirten Seite compensirt ist.

1) 1. c. 238 . 
Endlich sei hier auch auf die interessanten Versuche Kre i d l's ${ }^{1}$ ) an Krebsen mit "eisernen" Otolithen hingerviesen. Diese Thiere zeigen gegen die Einwirkung des Electromagueten folgendes charakteristische Verhalten : „wenn man den Magnetpol einer Otocyste von seitlich und oben nähert, so bleibt das Thier, so lange kein Strom durch den Electromagneten geht, vollkommen ruhig; in dem Momente, wo man den Strom schliesst und der Stab zu einem Magneten wird, dreht sich das Thier vom Magneten weg, so dass die Medianebene seines Leibes geneigt ist, und zwar entfernt sich dieselbe um so stärke: vom Magneten weg, je näher man an das Thier herankommt."

Eine Erklärung für dieses Verhalten der Thiere dem Magneten gegeniber findet nun $\mathrm{Kreidl}$ nach einer mathematischen Analyse der auf die Otolithen wirkenden Kräfte (Zug- und Druckcomponente als Componenten der Schwerkraft) folgendermassen: Das Thier wird in Folge der Einwirkung des Magneten die Empfindang haben, nach der Seite geneigt zu sein, und es wird in Folge dessen, da es das Bestreben hat, seine normale Lage einzunèhmen, versuchen, sich gerade zu legen, was es nur thun kann, wenn es sich entsprechend stark nach der entgegengesetzten Seite neigt. (pg. 17 ff.)

Ueber das Auftreten der geschilderten Kopfverdrehungen lässt sich so viel sagen, dass dieselben die Folgen einer hochgradigen einseitigen Labyrinthverletzung sind. Sie sind nicht allein nach der eben beschriebenen einseitigen Herausnahme des ganzen Labyrinthes constante Erscheinungen, sie sind auch nach isolirter Herausnahme von zwei Ampullen beobachtet. Ganz ohne Einfluss scheint dabei die Entfernung der Schnecken zu sein, davon später!

\section{ß) Doppelseitige Totalexstirpationen.}

Welche hohe Bedeutung das Ohrlabyrinth für die Wahrnehmung der Kopfhaltung bei Tauben hat, geht am deutlichsten aus dem Verhalten der Thiere hervor, denen in einer Sitzung beide Labyrinthe vollkommen entfernt sind. Die dabei za beobachtenden Erscheinungen tragen deutlich den Charakter von Ausfallserscheinungen. Der Kopf wackelt wie ein Fremdkörper am

1) 1. e. 
Leibe. Bei manchen Thieren gehorcht er, wenn man den Körper in verschiedene Lagen bringt, anscheinend nur der Schwere, $d$. h. er sucht stets den tiefsten Punkt einzunehmen (cf. Ewald's Versuch 1).

An diese regellosen Kopfbewegungen schliessen sich die Störungen der Körperbewegungen an, gleichfalls ohne vorherrschende Richtungen, keine Verdrehungen, zuweilen Umdrehungen nach beiden Seiten abwechselnd, lediglich bestimmt durch die jeweilige abnorme Kopfbewegung.

Bei den normalen Tauben beobachtet man eine Art pendelnder Kopfbewegungen, die zur Aufrechterhaltung des Gleichgewichtes dienen und ohne Zweifel eine Folge der aus sämmtlichen normal funktionirenden Canälen ausgelösten Empfindungen sind. Ewald scbreibt dariiber: „die normalen Tauben zeigen einen merkwürdigen, übrigens bei sehr vielen Vögeln vorhandenen Reflex, den ich Kopf-Stoss-Reflex bezeichnen möchte. Bei jedem Schritt wird der Kopf gerade nach vorn gestossen und dann etwas langsamer wieder zurïckgezogen" (pg. 14). - Es handelt sich bierbei um Compensationsbewegungen zur Erhaltung des Gleichgewichtes. Die doppelseitige Entfernung der Labyrinthe vernichtet nun die normale Thätigkeit dieser Vorrichtungen. Werden die Thiere sich selbst überlassen, so beobachtet man, dass sie den (nun wackligen) Kopf in überstreckter Haltung durch krampfhafte Anstrengung der Nackenmuskulatur za fixiren trachten. Ewald schreibt: „Der Kopf wird beim Gehen gern etwas nach hinten getragen und der Schnabel nach oben gerichtet." Desgleichen wird die Wirbelsäule durcb die Anspannung der Rückenmuskeln nach hinten fixirt, um dem schwankenden Kopfe eine bessere Unterlage zu gewähren. Auf diese Weise erbält die ganze Haltung der Thiere etwas eigenthïmlich Steifes. Man kann das Verhalten dieser Thiere wohl vergleichen mit der ïberstreckten Kopfhaltnng eines Menschen, der sich bemüht, einen hohen Gegenstand, z. B. eine Pfauenfeder wie die Taschenspieler auf der Stirn zu balanciren. Die Tauben balanciren ihren eigenen Kopf, dessen Schwerpunkt durch die abnorme Haltung auf die durch Muskelkräfte fixirte Wirbelsäule verlagert wird.

Aus diesem Verhalten erklärt sich denn wohl die bekannte Unlust der Thiere zu irgend welchen Bewegungen und der Unwille, mit dem eine Ermunterung dazu beantwortet wird, sehr 
natürlich. Im Allgemeinen zwingt nur der Hunger dazu, die neue Gleichgewichtslage des Kopfes aufzugeben. Die Beobachtungen beim Fressen (Fressstörungen) zeigen in Folge dessen die Hülflosigkeit der Thiere am deutlichsten. Der Schnabel wird unter solchen Umständen ziellos nach dem Futter gestossen, hierbei gelingt es nur in den seltensten Fällen, ein Körnchen oder eine Erbse zu fassen. Und wenn es auch wirklich geglückt ist, so fliegt der Inhalt doch bei der Ausfibrung der Schleuderbewegung, die dazu dienen soll, die Nahrung mit Hülfe der Zunge nach dem Rachen zu befördern, wieder heraus. Bei dieser Bewegung verliert denn auch der Kopf jeden Halt, und es kommt zu turbulentem, regellosen Umherschleudern desselben, wobei denn natürlich. ebenso heftige, unregelmässige Körperbewegungen stattfinden. Ganz allmählich und nur unter den grössten Anstrengungen gelingt es den Thieren, den Kopf wieder in der bekannten überstreckten Haltung zu fixiren, um dann von Neuem den eben geschilderten Vorgang durchzumachen, da sie der Hunger dazu zwingt.

Da ist es denn wohl erklärlich, dass bei dieser fast unaufhörlichen Unruhe mit excessiven Muskelanstrengungen die Thiere abmagern und schliesslich zu Grunde gehen, wenn man nicht durch sorgfältige Ueberwachung der Nahrungsaufnahme dagegen Vorkehruugen trifft. Mein erstes derart operirtes Thier ist schliesslich an hochgradiger Atrophie, welche anch Ewald an einem Thiere beobachtet bat, gestorben. Weitere Versuchsthiere, bei denen die Ernährung sorgfältig überwacht wird, haben dagegen einen auffallend guten Ernährungszustand gezeigt, sodass ich F I o u re n s vollkommen beipflichten kann, der von seinen Thieren schreibt, sie wären gemästet gewesen. Auch $\mathrm{E}$ wald schreibt p. 21: „in späterer Zeit sah ich indessen die Thiere dauernd im besten Ernährungszustande verbleiben, und es ist jedenfalls sicher, dass eine solche Muskelatrophie nicht einzutreten braucht."

Nach Verlauf einiger Zeit haben denn auch meine Thiere wieder gelernt, selbständig Nahrung zu sich zu nehmen.

\section{r) Doppelseitige Exstirpation der Schnecken.}

Um diese sehr subtile Operation auszuführen, dringe ich nach Freilegung der canales horizontales und ihrer Sinus; um stets auf die Vermeidung einer Blutung zu achten, mit der grössten Vorsicht im vorderen unteren Quadranten des Bogengangkreuzes durch 
die feinen Knochenbälkchen in die Tiefe. Eine Blutung ist bei diesem Eingriffe so unangenehm, dass die Operation am besten abgebrochen wird. Liegt nun die Gegend der Ampullen der canal. horiz. und canal. vertic. post. ganz sauber frei präparirt da, so wird von hier aus nach vorne, unten und innen, also in der Richtung, wo die Schnecken liegen, vorsichtig das Knochengewebe entfernt ${ }^{1}$ ). Bei geniigender Vorsicht bleibt man dabei dem im Kreisbogen das nur wenige qmm grosse Operationsfeld umziebenden Sinus fern, indem man gut thut, die sicher geführte Nadel von unten her arbeiten zu lassen. Bei genügender Beleuchtung sieht man den Sinus durch das reine Knochengewebe stets durchsebimmern. Diesen Vortheil bebt die geringste Blutung in dieser Gegend sofort auf. Eine einwandfreie Operation ist dann wohl kaum zu erwarten.

Das Ausfliessen der lymphatischen Flüssigkeit gibt auch hier das Zeichen, dass man am rechten Orte ist, and nun gelingt es unschwer, durch eine genügend erweiterte Oeffnung ein feines Hälschen in das Lumen der Schnecke vorzuschieben, mehrmals umzidrehen und den Inbalt herauszubefördern. Vermöge ihres Baues folgt die häutige Sihnecke fast immer in toto. Auch bei diesen Versuchen wird die mikroseopische Untersuchung des gewonnenen Gebildes stets vorgenommen.

Das Verhalten derartig operirter Thiere unterseheidet sich nun sehr auffallend von solchen Thieren, bei denen der Vestibularapparat verletzt ist. Wohl reagiren dieselben während der Operaration auf das Anbohren der Knochen und auf das Ausziehen der Schnecke durch einige Abwehrbewegungen - allein nichts von den auffallenden lebbaften Schmerzäusserungen noch den Kopfbewegungen, deren Heftigkeit bei den geringsten Eingriffen auf die pars sup. des Ohrlabyrinthes sofort imponirt. Auch nach der Operation zeigen die Thiere keinerlei Störungen weder in der Kopfhaltung noch in den Körperbewegungen: sie laufen wie gesunde Tauben umher, fliegen hoch empor, nehmen durchaus selbstständig Nahrung zu sich - kurzum, man merkt ihnen nichts Ab. normes an, trotzdem beide Schnecken entfernt sind.

1) Betreffs weiterer anatomischer Angaben sei hier auf Ewald's anatomische Bemerkungen hingewiesen, welche wohl für die Ausführung derartiger Operationen an Tauben hinreichend orientiren. 
Es entsprechen demnach diese Versuchsresultate an Tauben genau den Beobachtungen von Flourens an Kaninchen, denen er beide Schnecken isolirt entfernt hat, auch bei ihnen werden die eigenthümlichen Bewegungen, welche auf eine Verletzung der halbzirkelförmigen Canäle erfolgen, vermisst.

Bezüglich des Nachweises, dass derartig operirte Thiere noch Gebörsempindungen haben, sei auf $\S 3$ verwiesen.

\section{\$ 3. Theoretisches und Kritisches.}

Am Schlusse seines umfangreichen. Werkes fasst $\mathrm{E}$ wald die Ergebnisse seiner physiologischen Untersuchungen über das Endorgan des N. octavus zusammen. Das Labyrinth bestebt bei den böberen Wirbelthieren aus

I. dem "Tonuslabyrinthe", welches den Ohrtonus der Muskulatur unterhält. Indem es anch als Sinnesorgan functionirt, zerfällt es in

a) das Goltz'sche Sinnesorgan (Bogengangapparat),

b) die Maculae acusticae (Otolithenapparat),

II. dem Hörlabyrinthe.

Ad. I. Die ersten von Professor E w a l d geäusserten Tonusanschauungeu scheinen mir in einem Zusammenhange mit den gleichfalls von Strassburg aus veröffentlichten anatomischen Untersuchungen $K \ddot{o p} p$ e $\mathrm{n}^{\prime} \mathrm{s}^{1}$ ) ïber die Anatomie des Froschgehirns zu stehen. E w al d erwähnt diese Arbeit an zwei Stellen mit kurzen Worten. Köp p e n macht auf interessante Beziehungen zwischen dem sog. Acusticusfelde und den etwa den Vordersträngen des Säugethierrückenmarkes entsprechenden Ventralsträngen anfmerksam. Diese Stränge, zeichnen sich durch besonders starke Fasern aus ("Grossfaserbündel“). Er knüpft an diese Befunde folgendermassen an: „Nach allen den angeführten Thatsachen möchte ich folgende Hypothese aufstellen. Jene Gruppe grösster Zellen (im Acusticusfelde gelegen) ist das Centrum für die Grossfaserbündel, die also nach der einen Seite mit den M üller'schen Fasern, nach der anderen Seite mit den stärksten Fasern des Acusticus verbunden sind, in der Weise, dass aus einom Theil jener Zellen die Grossfaserbündel entspringen, aus einem andern die starken

1) Arch. f. Anat. u. Physiol. (anat. Abth.) 1888. 
Acusticusfasern. Würde es nun noch möglich sein zu beweisen, dass die stärksten Acusticusfasern den halbzirkelförmigen Canälen zugingen, so wäre es weiter denkbar, dass in den grossen Zellen ein Gleichgewichtscentrum läge, welches, wie ich bei Besprechung des Rückenmarkes gezeigt habe, mit den motorischen Wurzeln zusammenhängt, durch die Acusticnsfasern audererseits mit den halbzirkelförmigen Canälen. Es sei nun gleich bemerkt, dass möglicherweise auch die stärksten Fasern direct in den Acusticus iibergehen und ferner auch, dass von den grossen Zellen aus nicht starke Fasern, sondern feine Fortsätze, diese mit den halbzirkelförmigen Canälen verbinden könnten."

E w a 1 d fasst nun offenbar diese Fasern als sog. Tonusfasern anf und nimmt seither an, „dass vom $\mathrm{Ohr}$ aus beständig ein Tonus der Muskulatur angeregt wird und dass der Fortfall dieses Tonus auch das Muskelgefühl schädigt etc." ${ }^{1}$ ).

Die Störungen, welche von dem Tonuslabyrinthe ansgehen können, bestehen in einem Mangel an Präcision beim Gebrauche der quergestreiften Muskulatur und haben sämmtlich den Charakter von Ausfallserscheinungen. Am meisten betroffen werden die am prä eisesten arbeitenden Muskeln, ihrer Reihenfolge nach also etwa zuerst die Augenmuskeln, dann die Halsmuskeln, Flügelmuskeln und endlich Beinmuskeln. Die Störungen sind nun nicht nur relativ, sondern auch absolut um so grösser, je mehr Präcision bei den normalen Bewegungen angetroffen wird. Ewald hat nun iiber die Wirkung auf die einzelnen Muskelgruppen bis jetzt etwa Folgendes feststellen können: „Jedes Labyrinth hängt vorzugsweise mit den Muskeln der gekreuzten Körperseite zusammen, welche die Wirbelsäule und den Kopf bewegen (Nackenmuskulatur, Halsmuskulatur und namentlich die Wirbelmuskeln, welche vom Körper des unteren Wirbels zu den Querfortsätzen der oberen gehen). Bei den Muskeln der Extremitäten ist eine Theilung zwischen den Streckern und Abductoren einerseits und den Beugern und Adductoren andererseits vorhanden. Jedes Labyrinth ist mit den ersteren der gleichen Körperseite und mit den letzteren auf der gekreuzten Seite enger verbunden. Alle Augenmuskeln mit Ausnahme des Musculus rectus ext. scheinen hauptsächlich von dem benachbarten

1) Bedeutung des Ohres für die normale Muskelcontraction. Centralbl. f. Physiol. Bd. V. 1891. 
Labyrinthe abzuhängen“ (p. 297). Die Folge dieser Vertheilung ist die spiralige Krümmung der Wirbelsäule nach der operirten Seite, ebendahiu sind Kopf und Hals geneigt, die Extremitäten derselben Seite sind flectirt und adducirt, die der gekreuzten gestreckt und abducirt, die Augen, besonders das benachbarte, nach der operirten Seite hin abgelenkt.

Das Labyrinth tibt nun einen bestïndigen "Ohrtonus". ans. Diese Thätigkeit kann gehemmt und gesteigert werden, was einmal aus den Störungen nach Fortnabme der Labyrinthe hervorgeht, ferner aber aus der doppelten Wirkung der electrischen Ströme, der passiven Rotation oder des künstlich erzeugten Endolymphstromes zu schliessen ist, indem stets zwei versehiedene und zwar entgegengesetzte Bewegungen des Thieres beobachtet werden. Wie die beständige Erregung der Octavusfasern auf die Muskeln wirkt, muss vorläufig dahingestellt bleiben.

Welcher Art sind nun die beständigen Erregungen? Ewald trennt zunächst die mit Haaren versehenen Zellen in hörhaaretragende (Octavusenden in der Schnecke) und tonushaaretragende (cristae ampullarum und maculae acusticae). Ein beständiger Endolymphstrom kann nun aus verschiedenen Gründen nicht diese Haare in beständiger Bewegung erhalten. Dagegen können sich "die Tonushaare activ bewegen". $\mathrm{H}$ as s e hat ja bereits bei vielen Thieren flimmernde Haare im Gehörorgan gefunden, selbst bei einem Wirbelthiere. Die Tonushaare würden also die Endolymphe in einer bestimmten Richtung fortbewegen, welche Bewegung durch andere Kräfte beeinflusst, die Thätigkeit der Tonushaare unterstiitzen oder erschweren kann. - Das Tonuslabyrinth ist aber als Sinnesorgan anzusehen, denn es wird in seinen Ampullen wenigstens, vielleicht in ganzer Ausdehnung durch die Drehungen des Kopfes beeinflusst und vermittelt eine Wirkung der letzteren je nach ibrer Richtung und Stärke auf den Körper. Dieser "Sinn“ kann nicht unter die Rubrik der Tastempfindungen gerechnet werden. Wir können auch die durch die Octavuserregungen vermittelten Wahrnehmungen nicht nach anssen verlegen, also nicht lokalisiren. Wir haben es demnach mit einem neuen, dem sechsten, dem Goltz'sehen Sinne ${ }^{1}$ ) zu thun.

1) Vgl. He n s e'n, Vortrag gegen den sechsten Sinn. Arch. f. Ohrenheilk. Bd. 35 . 
Wie bereits in der vorläufigen Mittheilung ${ }^{1}$ ) angemerkt ist, besteht noch eine Tonustheorie, welche von $\mathrm{Gad}^{2}$ ) aufgestellt ist. Derselbe sucht durch eine vorausgeschickte Analyse der pbysikalischen Vorbedingungen für die Wirkung der dureh die Schallschwingungen des Steigbuigels erzeugten Bewegungen der LabyrinthAlissigkeit nachuweisen, dass der Otolithenapparat des Vestibulums und die Hörhaare der cristae acusticae an diesen Bewegungen, insofern sie eine Gehörsempfindung anslösen könnten, nicht theilnehmen können. Diese Endapparate müssen also eine andere Function haben. Die Verbindung der zu ihnen gehörenden Nervenfasern mit dem Kleinhirn, welches erfahrungsgemäss keine Beziehung zu bewussten Sinneswahrnehmungen erkennen lässt, macht es $\mathrm{Gad}$ wahrscheinlich, dass es sich hierbei um Organe handelt, deren Function auf einer unbewusst reflectorisch sich vollziehenden Correction der Muskelinnervation im Interesse des Körpergleichgewichtes beruht. "Die Function dieses statischen Apparates berubt darauf, dass jeder Stellung und Haltung unseres Körpers periphere Sinneseindrücke in einer bestimmten Combination entsprechen, welche auf reflectorischem Wege einen zweckmässig abgestimmten Tonus von Körpermuskeln unterhalten und dass jede passive oder active Bewegung unseres Körpers odér seiner Theile mit einer Aenderung in der Combination der Sinneseindrücke verbunden ist, welche Aenderung reflectorisch zu einer der veränderten mechanischen Bedingung angepassten neuen Vertheilung der Spannungen oder auch zu corrigirenden Bewegungen führt. Das Kleinhirn ist nun durch seine Verbindung mit den peripherischen sensiblen Nervenendapparaten der äusseren Haut, der Sehnen. Gelenkenden der Knochen u. s. w., ferner durch die Aufnahme gewisser ebenfalls durch active oder passive Körperbewegungen veranlassten Bewegungsempfindungen der Netzhaut oder der Augenmuskeln wesentlich an den regulatorischen Vorgängen betheiligt. Da nun die Vestibularnerven des Ohres ebenfalls in das Kleinhirn führen, so ist auch zu erwarten, dass die auf dieser Bahn zugeleiteten Erregungen derselben Function dienen."

Um nun gegenuiber den vorstehenden Theorien seinen Standpunkt zu characterisiren, so wird von dem Verfasser in der

1) Fortschritte der Medizin. Bd. XI. 15. Febr. 1894.

2) Schwartze, Handbuch d. Ohrenheilk. 1892. 
Hauptsache die mangelhafte (nach einseitigen Exstirpationen) Functionirung des statischen Sinnes oder dessen gänzlicher Fortfall (nach doppelseitiger Exstirpation) als Ursache der geschilderten Bewegungs stör ungen angesehen.

Bei den durch Ueberwachung der Ernährung im besten ' Zustande erhaltenen doppelseitig labyrintblosen Thieren ist ein einwandfreier Nachweis für das Bestehen eines Tonuslabyrinthes im Sinne der Ewald'schen. Theorie nicht gelungen. Oft haben z. B. vollkommen normale Thiere bei Versuchen mit Ausschluss der Orientirung durch die Augen mittelst einer Kappe die in Fig. 3 der Ewald'schen Untersuchungen abgebildete überstreckte Kopfhaltung viel besser gezeigt als jenes durch mangelhafte Nahrungsaufnahme zu Grunde gegangene erste Versuchsthier. Die späteren Versuche haben derartiges nicht mehr erkennen lassen.

Beziiglich der Gad'schen Tonustheorie lässt sich wohl nur sagen, dass sie einen Erklärungsversuch der Wirkung des statischen Sinnes darstellt. Auf eine Besprechnng der physikalischen Betrachtungen und ihrer Resultate kann hier nicht näher eingegangen werden, die experimentellen Resultate sprechen dagegen.

Ad II. Die Function des Hörlabyrinthes ist bekannt. Eine besondere Besprechung erheischt die Behauptung, dass nicht nur der Endapparat, sondern auch der Acusticusstamm durch Schallwellen erregbar sei. Und zwar sollen nach $\mathrm{Ewald}^{1}$ ) die beiderseits labyrinthlosen Thiere bei Anwendung bestimmter Vorsichtsmassregeln (Ausschliessung der Einwirkung von Tastreizen) noch alle lauten Geräusche hören und alle Töne bis zum zweigestrichenen $a$, also bis zu 1000 Schwingungen in der Sekunde. Die tiefen Töne würden besser wahrgenommen als die höheren. Dass sie diese Gehörswahrnehmungen gehabt haben, zeigen die Thiere, indem sie "reagiren". „Es müssen also die Schallwellen den Stamm des Octavus erregen und auf diese Weise die Gehörsempfindung vermitteln" (p. 26). Dagegen werden dic Tauben stocktaub, wenn die ,Octarus-Aeste" mit kleinen Haken ausgebohrt werden und dann in die Knochenöffnungen Spuren einer Arsenikpaste eingebracht wird ${ }^{2}$ ).

1) Vgl. Fano und M assini. Centralbl. f. Physiol. Bd. IV. Nr. 25.

2) Vgl. Hensen's treffende Einwendungen 1. e. 
Worin die „Reactionen" bestanden haben, darïber erfahren wir von Ewald nichts. Jedenfalls hütet sich dieser Autor mit Recht vor der Angabe etwaiger Kopfbewegungen, aus denen auf die Gehörswahrnehmungen geschlossen werden möchte. Wohl bedacht handelt es sich bei den $z u$ prüfenden Thieren um solehe, denen durch Herausnahme beider Labyrinthe das statische Sinnesorgan, welches die Wahrnehmung der Kopfhaltung vermittelt, genommen ist. Jedwede Kopfbewegung ist demnach mit der grössten Vorsieht zu deuten.

Um nun seine Entdeckung durch eine Autorität bestätigen und sichern zu lassen, schickt $\mathrm{E}$ wald eine doppelseitig labyrinthlose Taube an $\mathrm{W}$ und nach Leipzig mit der Bedingung; wenn sich Wundt von dem noch vorhandenen Hörvermögen đes Thieres uiberzeugt haben sollte, auch selbst persönlich dieser Ueberzeugnng irgend einen öfentlichen Ausdruck zu geben. Dies geschieht denn in der That durch ,Acustische Versuche an einer labyrinthlosen Taube von $W$. Wundt"1).

Hier werden denn nun durch Tabellen die Versuchsreihen illustrirt, die an diesem Thiere und meist gleichzeitig an einem normalen angestellt sind. Die acustischen Reize haben theils in den Klängen eines Harmoniums und zwar ebensowohl in Einzelklängen, wie in verschiedenartigen Zusammenklängen, theils in Geräuschen von verschiedener Beschaffenheit, wie Klopfen an der Thïre, klatschende Geräusche, electrische Klingeln und dgl. bestanden. Folgende kleine Tabelle soll eine Uebersicht über die angestellten Versuche und ihre Resultate wiedergeben. Die Aus driicke, betreffend die Resultate, sind aus dem Original entlehnt. In 9 Versuchsreihen reagiren folgendermassen :

\begin{tabular}{|c|c|c|c|c|}
\hline & $\begin{array}{l}\text { die oper } \\
\text { Klänge }\end{array}$ & $\begin{array}{l}\text { rte Taube } \\
\text { und zw } \\
\text { Geräusche }\end{array}$ & $\begin{array}{l}\text { die norn } \\
\text { ar auf } \\
\text { Klänge }\end{array}$ & $\begin{array}{l}\text { ale Taube } \\
\text { Geräusche }\end{array}$ \\
\hline 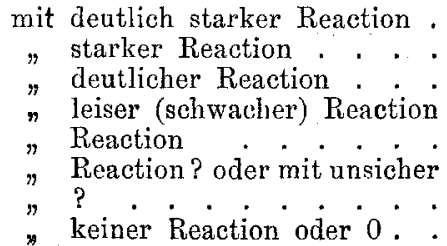 & $\begin{array}{r}2 \\
9 \\
3 \\
- \\
- \\
5\end{array}$ & $\begin{array}{r}- \\
10 \\
3 \\
1 \\
1 \\
2 \\
19\end{array}$ & $\begin{array}{r}1 \\
1 \\
5 \\
14 \\
5 \\
1 \\
20 \\
23\end{array}$ & $\begin{array}{l}- \\
7 \\
8 \\
2 \\
3 \\
1 \\
3\end{array}$ \\
\hline
\end{tabular}

1) Philosophische Studien. IX. Bd. 4. Heft. 
Das Versuchsresultat besteht nach $W u n d t$ darin: „Das einzige, was sich mit Bestimmtheit sagen lässt, ist: die labyrinthlose hat genau ebenso wie die normale Taube auf die meisten Schallerregungen reagirt; bei beiden ist dies in solcher Weise geschehen, dass eine Täuschung durch zufällige spontane Bewegungen ausgeschlossen ist, und bei beiden zeigen diese Reactionsbewegungen im Wesentlichen die nämlichen Merkmale wie die, aus denen wir bei unseren hörenden Mitmenschen schliessen, dass sie wirklich hören. Demnach werden wir auch mit demjenigen Grad von Sicherheit, der uns überhaupt bei Aussagen über subjective Erlebnisse anderer Wesen auf Grund der an ihnen beobachteten objectiven Symptome zu Gebote steht, schliessen dürfen, dass die labyrinthlose Taube wirklich gehört habe; und da durchaus nicht bekannt ist, dass es andere Sinnesorgane oder andere Sinnesnerven giebt, die Gehörsempfindungen vermitteln, ausser dem Acusticus, so scheint die Annahme geboten, dass in diesem. Falle die Schallwellen durch ihre directe Einwirkung auf "die noch erhaltenen Acusticusfasern" die Schallperceptionen veranlasst haben" (p. 505).

Die Operation war in Strassburg auf der rechten Seite am 26. April 1893, auf der linken am 30. Mai ausgeführt worden. Die Trommelfelle sind beiderseits am 3. Juli zerstört worden, „um dem Einwande, die Thiere könnten die Schwingungen des Trommelfelles als Tastreize empfinden, von vornherein zu begegnen $\left.{ }^{1}\right)^{\text {“. }}$. Die Schallprïfungen geschahen in Leipzig vom 17.-31. Juli, an welchem Tage Dr. HeId die Section des getödteten Thieres vorgenommen hat. Dabei findet sich, dass erstens die Operation von $\mathrm{E}$ w a l d tadellos ausgeführt worden ist. Die microscopische Untersuchung kann keine nervösen Gebilde nachweisen. Daneben hat sich aber auch gefunden: ,der Hörnerv war beiderseits nur noch als ein dünner Faden sichtbar. Das Tuberculum acusticum war stark atrophisch, von grauem Aussehen. Ebenso waren die Furchen des Kleinhirns etwas atrophisch und daher keilförmig gestellt. Auch der linke Occipitotemporallappen des Grosshirns zeigte stärkere, der rechte nur schwache $A$ trophie ${ }^{\star}$ (p. 504).

1) Diese Bemerkung ist nicht stichhaltig, da die grosse Regenerationsfähigkeit der Trommelfelle allgemein bekannt ist. Bis zum Abschluss der Versuchsreihen, also nach etwa 4 Wochen, ist zumal bei dem bekannten schnellen Verlaufe der Heilungsprocesse bei Tauben sicher eine vollkommene Regeneration vorhanden gewesen.

E. Pfinger, Archiv f. Physiologie. Bd. 57 . 
Den vorstehenden Versuchen von Ewald und $W$ undt gegenüber muss ich hier zunächst, wie es bereits in der vorläufigen Mittlieilung geschehen ist, nochmals betonen, dass deutliche Ge hö rsreaction en bei Tauben nur schwierig zu erzielen sind. Alle Versuche mit Stimmgabeln, Klingeln, Pfeifen und sonstigen Tönen oder Geräuschen haben nicht einmal an normalen, zur Controle dienenden Thieren eine constante Reaction erzielen können. Ferner muss man eine mehrfache Wiederholung derselben oder ähnlicher Schallreize vermeiden, die Thiere -- selbst die wenigen, die sich überhaupt zu derartigen Versuchen eignen - werden sehr bald selbst gegen die stärksten Reize theilnahmlos. Ausserdem spielen äussere Umstände, wodurch ihre Aufmerksamkeit beeinflusst werden kann, eine Rolle.

Wie richtig diese Beobachtungen sind, geht sehr dentlich ans W und t's Versuchstabellen (of. Nr. 10!) hervor. Und wenn aus ihnen gefolgert wird, dass die labyrinthlose Taube genau ebenso wie die normale anf die meisten Schallerregungen ,reagirt" hat, so lässt sich mit vielleicht noch mehr Recht daraus der Schluss zieben, dass die laby in thlose Taube noch besser gehört habe als die normale! Es geht wohl daraus die Unzuverlässigkeit und Unvollkommenbeit der angewandten Prüfungsmethoden deutlich hervor.

Bei allen diesen Schwierigkeiten habe ich mich denn auf die reflectorische Schlussreaction der Hauptsache nach beschränkt. Die Prüfungen werden zu gleicher Zeit an gesunden Thieren angestellt. Die Gesichtswahrnehmungen sind durch Lederkappen ausgeschlossen. Sobald sich nun die Thiere beruhigt baben, was immerhin einige Zeit dauert, sieht man, dass die gesunden Thiere beim Abschiessen einer Zimmerpistole erschreckt zusammenknicken, während sich die beiderseits labyrinthlosen Tauben vollkommen ruhig verhalten.

Man kann denselben Versuch auch derart veranstalten, dass man die Thiere mit beiden Händen auf einer ebenen Fläche (Tischplatte) festhält, so dass sie keinerlei Bewegungen mit dem Rumpfe oder Extremitäten ausführen können. Wird dann ein Tuch iber den Kopf gedeckt, so tritt sehr bald eine Art hypnotischer Zustand ein. Vorsichtig kann man die Hände entfernen und ein anderes Thier genau ebenso daneben lagern. Werden auf diese Weise sowohl operirte als normale Thiere gleichzeitig der Prifung 
unterzogen, so tritt beim normalen Thiere auf Abschiessen der Pistole sofortiges Erwachen und Aufspringen ein, während die beiderseits labyrinthlosen Thierewiederumstill li egen bleiben.

Ausser diesen für etwaige Demonstrationen verwendbaren Prüfungen giebt es bei der langen Beobachtungszeit oft genug noch Gelegenheit, wo man sich den Thieren (beim Fressen, Einschlafen etc.) ungesehen nähern kann, um sich trotz Anwendung sehr starker Töne und Geräusche durch die Reactionslosigkeit von ihrer Taubheit zu überzeugen. Zufällige Gesichtswahrnehmungen oder auch nur leises Anblasen erwecken sofort die volle Aufmerksamkeit und damit entsprechende Reactionen.

Bei dieser Gelegenheit soll denn hier auch die im zweiten Theile bei der Schilderung der Thiere nach doppelseitiger Extraction der Schnecken weggelassene Gehörsprüfung aufgenommen werden. Es bedarf das nur des Zusatzes, dass in solchen Fällen statt zweier Thiere (normale und doppelseitig labyrinthlose) jedesmal die doppelseitig cochlealosen Tauben hinzugezogen worden sind. Die reflectorische Schussreaction tritt bei solchen Thieren durchaus präcis ein, aber es scheint doch eine Verminderung der Reactionsbewegungen unverkennbar. Zur Zeit tritt diese Reaction (Monate lang nach dem letzten Eingriffe) durchaus noch ein bei einem Thiere, bei dem zu gewissen Zwecken erst auf einer Seite eine Totalexstirpation vorgenommen ist, späterhin die Schnecke der andern Seite extrahirt und schliesslich vom canal. vertic. ant. membr. ein circa $1 \mathrm{~cm}$ langes Stück resecirt worden ist. Dasselbe Thier zeigt also neben hochgradigen Kopfverdrehungen, bei lebhafteren Bewegungen oder in Angstzuständen etc., die seit Januar d. J. bestehen, deutliche Reaction auf grobe Geräusche.

Bei Ewald's Angaben vermisse ich irgend welche $\mathrm{Zeit}$ angaben, in welcher nach der Operation noch ,dentliche Gehörsreactionen" nachgewiesen werden können. Und doch sind dieselben für die Beurtheilung der ganzen Streitfrage, ob die doppelseitig labyrinthlosen Tauben noch hören können oder nicht, von der grössten Wichtigkeit.

Die von $W$ undt geprïfte Taube, welche sogar noch auf Klänge „reagirt" hat, ist, als sie diese "Reactionen" gezeigt hat, vor ungefähr 12 resp. 6 Wochen operirt gewesen. Die Section erwies die vollständige Entfernung aller nervösen Elemente der 
Ohrlabyrinthe und einen beiderseits nur noch als diunnen Faden sichtbaren Hörnerven, ein ,stark atrophisches Tuberculum acusticum, von grauem Aussehen" ete.

Die allerdings recht mühevolle microscopische Untersuchung der Gehirne und Gehörorgane meiner sämmtlichen Versuchstbiere hat nun ergeben, dass bereits nach $2-3$ Wochen post operationem eine ausgebreitete secundäreaufsteigende Degeneration der Acusticusfasern bis zu den centralwärts gelegenen Kernen eingetreten ist. (Methode von Marchi und Algeri.) Für die Aufrechterhaltung der Ewald'schen Behauptung, die eine Stütze in Wundt's Versuchen gefunden hat, bliebe infolgedessen nur die Annahme ïbrig, dass auch degenerirte Nervenfasern durch Schallwellen erregt werden könnten. Die (geschilderten) eigenen Versuche haben aber auch nach dieser Richtung negative Resultate ergeben. So selbstverständlich es allgemein scheint, muss dennoch diesen Behauptungen und Versuchen gegenüber hier ausdrücklich betont werden, dass nach beiderseitiger vollständiger Entfernung der Endorgane des N. acusticus totale Taubheit die Folge ist. Die nach W und bewiesene Hinfälligkeit des Gesetzes der specifischen Energie müsste für diese Fälle besser begründet werden, vorliufig bleibt das Gesetz sonach wohl noch bestehen!

Bevor wir auf die mit Hilfe der Marchi-Algeri'schen Methode gewonnenen Resultate näLer eingehen, sollen einige einleitende anatomische Bemerkungen vorausgeschickt werden.

Der Nervus acusticus besteht bekanntlich aus zwei Aesten, die bisher gewöhnlich als vorderer und hinterer untersehieden worden sind. Bei Tauben theilen sich beide Aeste vor ihrem Eintritt ins Labyrinth in mehrere Zweige, welche durch bestimmte Foramina zu ibren zugehörigen Endapparaten ziehen.

Folgende tabellarische Uebersicht nach Retzius-Ewará wird am schnellsten orientieren:

a) der ramus anterior theilt sich in 3 Zweige:

1. Ramulus ampullae canalis vert. ant. zur dureh d. foram racrista acust. amp. ant.

muli ampullae ant.

2. „ ampullae canalis horizontalis zar crista acust. amp. horiz.

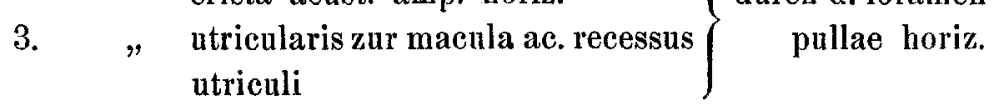


b) der ramus posterior theilt sich in 5 Zweige:

$\left.\begin{array}{c}\text { 1. Ramulus saccularis zur macula ac. } \\ \text { sacculi }\end{array}\right\} \begin{gathered}\text { durch d.foram. ramuli } \\ \text { sacculi. }\end{gathered}$

2. $"$ ampullae canalis vert. post. zur

crista ac. ampullae post. $\quad$ durch $d$. foram. am3. " $\begin{aligned} & \text { neglectus zur macula ac. ne- } \\ & \text { glecta }\end{aligned}$

4. " basilaris zur papilla ac. basil. J durch d. for. ramuli

5. " lagenae " ", lagenae $\}$ cochlearis.

Nach den neueren anatomischen Werken (vgl. Kölliker, Rauber u. A.) scheint aber die Trennung der beiden Bestandtheile des Acnsticus in den Nervus vestibularis und $\mathrm{N}$. cochlearis mit Recht vorgezogen zu werden. Wir schliessen uns daher dieser Aenderung an. In der obigen Tabelle sind in Folge dessen entsprechende Veränderungen vorzunehmen.

Beide Nerven-N. vestibularis und N. cochlearis - entspringen nun aus zwei.gleichnamigen Ganglien, nämlich aus dem Ganglionvestibulare und dem Ganglion cochleare. Diese Ganglien stellen aber nicht nur die histogenetischen, sonderna uch die Ernährungseentrafúr die zugebörigen Nerven dar. Die Lage dieser Ganglienzellenhaufen ist eine verschiedene, was für uns von besonderer Wichtigkeit ist ${ }^{1}$ ). Während nämlich ersteres, das Ganglion vestibulare, vom Labyrinth aus $d$. h. von aussen nicht zu erreichen ist, ohne die innere Ohrkapsel, die das Gebirn schïtzend bedeckt, aufzubrechen, liegt letzteres, das Ganglion cochleare, un ter der Nervenenda usbreitung in der häutigen Schnecke selbst.

Letzeres ist demnach bei einer von aussen her vorgenommenen vollkommenen Extraction der häutigen Schnecke, so dass man den Stumpf des N. cochlearis erkennen kann (Ewald), unmittelbar mit zu entfernen.

Beide Ganglien verhalten sich nun anatomisch wie $S$ p in a lganglien. Sie senden einen (peripheren) Fortsatz (4 in nachstebender Figur) nach den Neuroepithelien (1-2) im Labyrinth und einen zweiten (centralen) Fortsatz (6) nach der Medulla oblongata, der mit seinen Endbäumchen und Collateralen (8) zu den entsprechenden Kernen in Beziehung tritt. Diesen anatomischen

1) S. Abbildung I, sowie die zugehörigen Bemerkungen. 
Verhältnissen entsprechend müssen die Degenerationsbilder nach Exstirpation einzelner Theile oder des ganzen Labyrinthes ausfallen. Eine secund äreaufsteigende Degeneration

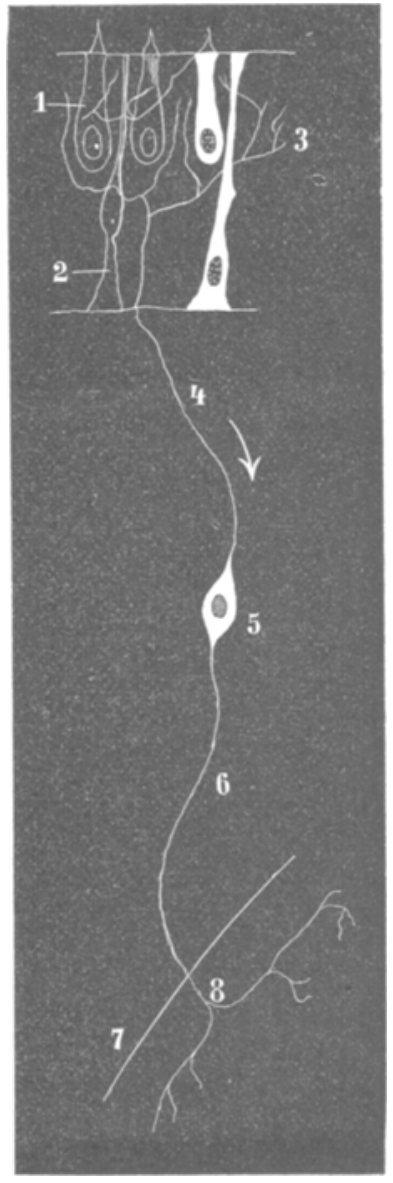

Schema der peripheren Endigung des $\mathbf{N}$. acusticus von Retzius, Biolog. Unter. suchungen Bd. IV, 1892. 1-2 Neuroepithel, 1 Haarzelle, 2 Faden- oder Stützzellen, 3 peripheres interepitheliales Endbäumchen, 4 Nervenfaser, 5 Zelle des Ganglion acusticum, 6 Neurit, 7 Grenzlinie des Gehirnes, 8 Theilung in einen auf- und einen absteigenden Ast, mit Collateralen derselben. Nach Rauber, Lehrb. der Anatomie 1894. des N. cochlearis mus dem. nach stets nach Ablaufeiner entsprechenden Zeit nacl vollständiger Entfernung der häutigen Schnecke, sodass der Nervenstumpf siehtbar ist, die Folge sein.

Anders verhält es sich mit dem $\mathrm{N}$. vestibularis und seinen peripheren Fasern. Hier soll man eigentlich erwarten, dass nach Ausräumung der Endapparate dieses Nerven des in die centripetale Bahn zwischen geschalteten Ganglion vestibulare wegen gar keine secundären Veränderungen eintreten können. Dies trifft aber nur zum Theil zu, indem doch jedesmal nach isolirter Exstirpation auch nur einzelner Theile des Vestibulums doch stets eine nicht unbeträchtliche Anzahl degenerirender Nervenfasern aufgefunden worden ist. Die Constanz der Erscheinung lässt vermuthen, dass es sich hierbei um gewisse durch das Ganglion durchziehende F asern handele, deren Zellen vielleicht den Character versprengter Ganglienzellen tragen. $\mathrm{V}$ i e $11 \mathrm{e}$ i $\mathrm{c} \mathrm{h} t$ aber haben wir es auch hier mitan der Peripherie gelegenen Sinnesepithelz e 11 e n zu thun, aus denen diese Fasern entspringen. (Vgl. Epithel der regio olfactoria, der Retina.) Hierbei sei denn auch gleich erwähnt, dass bei einigen Präparaten eine deutliche Verfolgung degenerirender Nervenfasern auf ihrem Wege durch das Ganglion vestibulare hindurch direkt bis ins Klein- 
hirn, die sich anscheinend in der Mittellinie kreuzen, möglich gewesen ist. Da die bisherigen Resultate noch keinen abschliessenden Charakter tragen können, so ist das genauere Studium dieser Verhältnisse späteren Untersuchungen zu überlassen.

Die von den Ganglien centripetal ziehenden Fasern des N. vestibularis und $\mathrm{N}$. cochlearis entsprechen den "Wurzeln" des $\mathrm{N}$. acusticus. Beim Säugethiere und beim Menschen stellt der N. cochlearis die laterale, untere oder hintere Wurzel dar, der N. vestibularis die mediale, obere oder vordere Wurzel. „Diese beiden Theile werden bei Vörgeln noch nicht durch das corpus restiforme getrennt, da dasselbe in dieser Gegend noch nicht soweit dorsalwärts gerïckt ist, vielmehr wird es durch die Vestibularis-Fasern durchbrochen." (Brandis.)

Zwischen den Nervenfasern beider rami findet nun eine umfangreiche Durchkreuzung statt. Die der Lage der Schnecke nach mehr von vorn herziehenden Fasern des N. cochlearis treten bauptsächlich mit Kernen in Beziehung, die mehr caudalwärts im verl. Marke liegen. Ausfibrlich kann hier nicht auf die anatomischen Verhältnisse des Vogelhirns eingegangen werden, es sei daher noch einmal auf die einschlägigen Arbeiten lingewiesen, speciell auf die zuletzt ersehienenen Untersuchungen ïber das Gehirn der Vögel von F. Brand is ${ }^{1}$ ), (Friedrichsberg, Hamburg). Ich kann diese Angaben für das Taubengehirn durchaus bestätigen. Nur darin gebt dieser Autor fehl, dass er den N. cochlearis aus einem Ganglion entspringen lässt, das „bedentend modificirt (gegeniiber dem Vestibularisganglion) ist und sich mit dem Hirnstamme gänzlich vereinigt hat." $\mathrm{Er}$ hat eben das in der Schnecke selbst gelegene Ganglion cochleare vernachlässigt und sein "Cochlearisursprung" bilden Kerne, die den Anfang der zweiten, cerebralwärts verlaufenden Nervenbahn (Neuron II) bedeuten. Der N. cochlearis endigt demnach, indem "seine durch ibre Stärke auffallenden Fasern im dichtgeschlossenen Complex an der dorsalen Peripherie und auch dem lateralen Rande mehr oder weniger genähert in die Med. obl. eindringen, an ziemlich grossen zwischen den Fasern auftretenden Zellen." Und hier endigt auch in den nach der Marchi'schen Methode gewonnenen Degenerationspräparaten die Hauptmasse der charakteristischen schwarzen Schollen, die im Be-

1) Arch. f. microscopische Anat. Bd. 42 u. 43. 
reiche dieser Kerne eine so starke Anhäufung zeigen, dass die Zellen wie überschïttet erscheinen. ( $\mathrm{ggl}$. dazu die Abbildungen II und III und deren Erklärungen.)

Man kann mehrere, mindestens zwei, mehr gesonderte Kerne trennen. Einen umfangreichen, länglichen, an der dorsalen Peripherie des Querschnittes gelegenen "grosszelligen" Kern und eine mehr lateral und nach aussen vom Cochlearisstamme gelagerte $\mathrm{Ab}$ theilung, gleichfalls von grossem Umfange, der "Eckkern“ (Brandis). Die Lage dieser Kerne erscheint bei verschiedenen Schnittführungen naturgemäss verschieden. Zwischen den "grosszelligen" Kernen beider Seiten stellt hauptsächlich der "Bogenzug" (wahrscheinlich den Striae medullares entsprechend) eine Verbindnng und zugleich eine eentrale Fortsetzung dar. (Vgl. Held's Schema der centralen Gebörleitung.)

Ventral vom Cochlearisstamme treten die Fasern des N. vestibularis in die Medulla obl. ein, indem sie die Fasern des corpus restiforme beim Eintritte fast rechtwinklig kreuzen. Weiterhin sind sie dorsalwärts und ziemlich stark proximalwärts gerichtet, so dass ihr Verlauf besser auf solehen Querschnitten zu übersehen ist, die schräg zur Längsachse und zwar in der Weise angelegt sind, dass die Mittellinie weiter cerebralwärts getroffen wird als die laterale Peripherio der einen Seite (Brandis). In einem Falle hat Brandi ${ }^{1}$ ) auch nach der von mir hauptsächlich angewendeten Methode ihre Faserrichtung darstellen können, nachdem einer Taube das Ganglion vestibulare experimentell entfernt worden ist. Nach drei Wochen haben die charakteristischen schwarzen Schollen den Verlauf der fast gänzlich zerfallenen Nervenfasern angezeigt. Leider schweigt der Autor über die Operationsmethode und das Verhalten des Thieres vollkommen.

Nach $\mathrm{Held}^{2}$ ) theilen sich die Achsencylinder des N. vestibularis bald nach ihrem Eintritte in das Gehirn in zwei Aeste. Bei den Vögeln richtet sich der eine derselben nach innen und zieht in der Richtung zur Raphe weiter, der andere wendet sich dorsalwärts zur Gegend des "kleinzelligen" Kernes oder direkt zum Kleinhirnschenkel und fasert sich hier bald auf ..... ${ }^{3}$ ). Auch

1) 1. c. Bd. 43. p. 110 .

2) Arch. f. Anat. u. Physiol. Anat. Abth. 1892,

3) Arch. f. microscopische Anat. Bd. 43. p. 110. 
Brandis nimmt an, dass sowohl der Nerv der Schnecke wie auch der des Vorhofes wahrscheinlich eine centrale Verbindung mit dem Kleinhirn zeigt. Es käme nun auch darauf an die Bedeutung der durchgehenden degenerirenden Fasern and ihre direkte Verbindung nach dem Kleinhirne zu ermitteln.

Die vergleichend-anatomische Betrachtung des Gebörorganes der Wirbelthiere deutet darauf hin, dass in der Entwicklung der Bestandtheile der pars superior und der pars inferior ein verschiedenes Verbältniss besteht. Letztere zeigt in der aufsteigenden Thier reihe fortgesetzt die Tendenz zu höherer Ausbildung und grösserer Vollkommenheit. Am deutlichsten tritt dies bei der vergleichenden Betrachtung ihres Hauptbestandtheiles, des ductus coehlearis, bervor. Derselbe besteht bei den Fischen als eine kleine Aussackung des hinteren Endes des Sacculus mit einem nervösen Endapparate, die Lagena mit der papilla acustica lagenae, auf der eine Otolitbenmembran gelegen ist.

Eine zweite Nervenendstelle besitzen die Amphibien, die papilla acustica basilaris. Letztere übertrifft nun bald bei höher entwickelten Thieren die erstere. Bei den meisten Sängern fällt dann die papilla lagenae ganz fort, während die papilla basilaris (spiralis) eine mächtige Entwicklung gewinnt.

Wenn wir dagegen die pars superior mit ihren Gebilden betrachten, so lässt sich dasselbe keineswegs nachweisen. Hier hat mit höherer Entwicklung der Thierreihe mindestens ein Stillstand der Entwicklung stattgefunden. Viele Thatsachen deuten aber sogar auf eine Art von Rü ckbildang hin nach gewisser Richtung wenigstens. Es sei beispielsweise auf einige Unterschiede zwischen den Gehörorganen der Vögel und denen der Menschen hingewiesen. Zunächst imponirt rein äusserlich der Bogengangapparat der Vögel hinsichtlich der Grösse und Ausdehnung der Formen, er übertrifft darin die entsprechenden Gebilde des Menschen. Der Form entsprechend gestalten sich auch die Verhältnisse der darin enthaltenen Gebilde. Bei Vögeln entspricht das Lumen der häutigen Bogengänge durchaus dem der knöchernen, beim Menschen zeigt der Querschnitt des knöchernen und häutigen Bogenganges einen so bedeutenden Unterschied, dass sich bei Betrachtung des kleinen, fast platt an die Wand gedrückten häutigen Bogenganges der Gedanke nicht 
abweisen lässt, es handele sich um einen Rückbildungsprocess. Auffälliger noch ist der Unterschied in der Ausbildung der nervösen Endapparate in den Ampullen. Dieselbe hat anscheinend bei den Vögeln die umfangreichste und eleganteste Ausbildung in dem Septum cruciatum in seiner mehr oder weniger vollkommenen Form erfahren. Im Vergleiche damit muissen die menschlichen eristae acusticae weit zurückstehen. Diesen Endapparaten entspricht wiederum die Anzahl der zugehörigen Nervenfasern und Ganglienzellen des Ganglion vestibulare. Brandis beschreibt das sehr grosse und auffällige Vestibularisganglion der Vögel, welches der hohen Entwicklungsstufe der Bogengänge in dieser Klasse entspricht. Mit diesem hervorragenden Gebilde bei Vögeln soll die Intumescentia ganglioformis Scarpae im meatus auditorius internus beim Menschen identisch sein, da wie $\mathrm{H}$ is jun. ${ }^{1}$ ) näher ausführt, das beim menschlichen Embryo noch vorbandene Ganglion vestibulare später zi diesem Gebilde wird ${ }^{2}$ ).

Bei diesen anatomischen Verschiedenheiten liegt der Gedanke nahe, dass auch die physiologisehe Bedeutungdieser $O \mathrm{rgane}$ bei den verglichenen Klassen $\mathrm{nicht}$ mehr die gleiche sein kann. Sicherlich hat das in der pars superior localisirte statische Sinnesorgan für die Vögel eine ungleich höhere Bedentung als fiir den Menschen. Ja selbst unter den einzelnen Vogelarten bestehen nach Brandis Verschiedenheiten. „Unterschiede in dem Baue des $\mathrm{N}$. vestibularis und in seinem Ursprunge sind bei den verschiedenen Ordnungen der Vögel kaum vorhanden, von Wichtigkeit ist nur die durch die Grösse des Ganglion und die Anzahl der Nervenfasern ausgedrückte stärkere oder schwächere Entwickelung; und diese scheint mehr auf die Lebensweise der Species als auf der inneren Verwandtschaft der Arten za beruhen. Die guten Flieger, wie Möve, Falke, Schwalbe, ganz besonders auch Cypselus, haben einen sehr starken Vorhofsnerv und übertreffen hierin die übrigen Vögel, unter denen die Hühnervögel am weitesten zuriuckstehen. Beim Strauss zeigt dieser Nerv eine in jeder Richtung sehr schwache Entwickelung." (Vgl. auch die Markscheidenentwickelung.)

1) Zur Entwicklungsgeschichte des Acustico-Facialisgebietes bein Menschen. Arch. f. Anat. u. Physiol. Anat. Abth. 1889. Suppl.-Bd.

2) Arch. f. microscopische Anat. Bd. 43. p. 113. 
Es leuchtet das auch vollkommen ein, wenn man bedenkt, dass bei den Fliegern während des Fluges an das statische Sinnesorgan die höchsten Ansprüche gestellt werden, indem bei der Bewegung in der Luft ein grosser Theil aller übrigen Vorrichtungen zur Erhaltung des Kopfgleichgewichtes sehr zurücktritt. Darauf kann denn auch die nach der Verletzung dieser Organe für lange Zeit bestehende Unmöglichkeit zu fliegen zurückgefübrt werden. Vielleicht spielen diese Organe auch bei dem Orientirungsvermögen der Vögel eine Rolle.

Beim Menschen kann wohl die statische Function der pars superior im Allgemeinen nicht derart hoch angeschlagen werden, dahingegen könnte die acustische Bedeutung diescr Organe wieder grösser geworden sein. In wieweit in Fällen von Erkrankung dieser Organe sich diese Verhältnisse verändern, darauf kann hier nicht eingegangen werden. --

Gegen die Existenz des statisehen Sinnesorganes im $\mathrm{Ohr}$ labyrinthe wird nach den letzten Untersuchungen, bei denen besonders die Ewald'schen Versuche eine hervorragende Rolle spielen, wohl Niemand mehr Einwendungen machen. Betreffs der Localisation sprechen Flourens' Resultate und die Ergebnisse der vorliegenden Experimente für die pars superior. Es hat sich nun nach doppelseitiger Entfernung der Schnecken herausgestellt, dass die Thiere auf oben geschilderte Gehörprüfungen in unzweideutiger Weise reagiren, demnach kommt der pars superior eine doppelte Function zu: erstens di e Wahrnehmung der Kopfhaltung im Sinne der arsprünglichen Goltz'schen Hypothese, zweitens aber auch Gehörse $m p$ find unge $n$ zu vermitteln.

Die pars superior enthält nun zwei, in ihrem Aufbaue verschiedene nervöse Gebilde, sie zerfällt nach Ewald in

a) das Goltz'sche Sinnesorgan (Bogengangapparat),

b) die maculae acusticae (Otolithenapparat).

Alle bisherigen Versuche deuten nun darauf hin, dass besonders die Bogengänge und Ampullen bei der statischen Function betheiligt sind. Es geht dies auch aus der vorhergehenden vergleichend-anatomischen Betrachtung deutlich hervor, indem gerade diese Organe, sobald anderweitige statische Vorrichtungen einer veränderten Lebensweise entsprechend ausreichend ausgebildet sind, einer regressiven Metamorphose anheim fallen. Den maculae acusticae 
käme demnach wobl die acustische Function za. Dafür spricht die Anwesenheit von Otolithen, die sich auch bei niederen Thieren in der Schnecke, dem Hauptorgan der acustischen Function, vorfinden. Nach dem, was man über die Leistungen der Schnecke bisher weiss, lässt sich wohl annehmen, dass die maculae nur der Perception der Geräusche dienen. Etwaige noch anzustellende experimentelle Nachweise für die verschiedenen Functionen in der pars superior dürften nicht leicht zu erbringen sein, unerlässlich würde ausserdem auch hierbei eine eingehende mikroskopische Untersuchung sein.

Hier soll dann noch, was in dem Vorstehenden genugsam begründet liegt, gegen die zunehmende Gewohnheit von den Otolithenorganen als im wesentlichen statischen Functionen dienenden Gebilden zu reden, Einspruch erhoben sein. Aus der vergleichendanatomischen Betrachtung geht dann noch klar hervor, dass mit den gesteigerten Ansprïchen an die Leistungen des Gehörorganes die Otolithen mehr und mehr verschwinden. -

Am Schlusse dieser Arbeit ist es mir ein Bedürfniss, meinem hochverehrten Lehrer, Herrn Prof. Dr. J. B e rnste in, für die wohlwollende Anregung zu diesen Untersuchungen und für die jederzeit gewährte Unterstützung bei der Ausführung derselben meinen tiefgefüblten Dank auszusprechen. Für das liebenswürdige Interesse und für die guitigst ertheilten Rathschläge bei der Anfertigung der Abbildungen bin ich ausserdem Herrn Geh. Medic.Rath Prof. Dr. Eberth sowie seinem Assistenten, Herrn Dr. med. R. B u g e, zu besonderem Danke verpflichtet.

\section{Nachtrag.}

Was die Anfertigung der Präparate anbetrifft, so werden die durch die Operation extrahirten Bestandtheile der Gehörorgane sofort zur Fixirung der nervösen Elemente in eine frisch bereitete $1 \%$ Osmiumsäurelösung anf 24 Stunden gelegt. Dann entweder in Glycerin ohne weitere Behandlung unter dem Mikroskop untersucht, oder, was meist geschehen ist, nach Abspülung in Wasser mit Alauukarmin gefärbt, mit Alcohol absol. gehärtet, in Celloidin eingebettet und geschnitten. In vielen Fällen wurden Serien angefertigt. 
Die Gehirne der Thiere werden zunächst anf $1-1 \frac{1}{2}$ Woche in reichlicher Menge $M$ ïller'scher Flüssigkeit gehärtet. Während dieser Zeit wird die Flïssigkeit mehrfach gewechselt und die Präparate in mehrere Scheiben zerlegt. Nur die nothwendigen Stïcke, Med. obl. sammt Kleinhirn, werden weiter behandelt, und zwar kommen diese nach Ablauf von $1 \frac{1}{2}$ Wochen in die $\mathrm{M}$ a rchi'sche Mischung (2 T. Müller + 1 T. 1\% Osmiums.), woselbst sie solange bleiben, bis sie eine dunkle Farbe angenommen haben. Man warte dazu ruhig 10-14 Tage bei gewöhnlicher Temperatur ab. Auch diese Mischung ist frisch bereitet, reichlich angewendet und gewöhnlich einmal erneuert worden. Dann werden die Präparate mit Alc. absol. nachgehärtet, in Celloidin eingebettet und unter $70 \%$ Alkohol in Serienschnitte von $0,02 \mathrm{~mm}$ Dicke zerlegt. Dieselben kommen direkt auf nummerirte Objectträger zu liegen, die vorher mit einer feinen Schicht wasserdünnen Celloidins übergossen sind. Ist ein Objectträger mit zweckmässig angeordneten Schnitten bedeckt - man thut gut, die Ränder der kurzen Seiten etwa $1 \mathrm{~cm}$ frei zu lassen -, so wird der überstehende Schneidealkohol mit Fliesspapier abgesogen und eine gleichartige Schicht Celloidins dariber gegossen. Nach kurzem Abdunsten kommen die Serien zum Entwässern einige Minuten in 90-96\% Alkohol und dann zum Aufhellen in Carbolxylol (Acid. carbol. cryst.: Xylol. puriss. $=1: 3$ ) und werden zum Schluss mit in Xylol gelöstem Damarharze mehrmals übergossen und ohne Deekglas auf bewahrt. Das ganze Verfahren ${ }^{1}$ ) ist an sich sehr einfach, iiber die Gefahren helfen einige Erfahrungen, die jeder machen wird, bald hinweg. Neben der von Marchi und Algeri angegebenen Methode ist auch die ron Weigert angegebene Markscheidenfärbung mebrfach angegeben worden, besonders hat dessen neueres Verfahren sehr gute Bilder geliefert.

Die zugehörigen Gehörorgane werden zunächst wie die extrahirten Labyrinthbestandtheile auf 24 Stunden mit $1 \%$ Osmiumsäure fixirt, dann in sehr reichlicher Menge $2 \%$ Chromsäure (Ranvier), der pro $100 \mathrm{ccm} 2 \mathrm{cem}$ reiner Salpetersäure (W a ldeyer) zugesetzt sind, entkalkt. Der ganze Process währt einige

1) Vgl. dazu: Edinger, nervöse Centralorgane. Leipzig 1892. p. 185 etc. und Friedländer-Eberth, microscopische Technik. 5. Auft. Berlin 1894. p. $89 \mathrm{ff}$. 
Wochen (3-4). Dann erfolgt die Härtung in Alcoh. absol., die Celloidineinbettung und die Anfertigung von Serienschnitten genau wie oben. Bei den meisten Präparaten wird ausserdem eine Färbung mit Hämatoxylin durchgeführt, die mit Hilfe einer der Differenzirflüssigkeiten (Weigert oder Pal) meist sehr schöne Bilder ergeben hat.

Die Schnittrichtungen sind sowobl bei den Gehirnen als bei den Gehörorganen verschiedene gewesen. Am häufigsten werden die Gehirne in Frontalschnittserien zerlegt, daneben sind aber auch Sagittalschnitte angefertigt. Bei den Gehörorganen sind Frontal- und Horizontalschnitte bevorzugt worden.

Es eribrigt vielleicht noch hier einige Angaben über die histologischen Verhältnisse im Labyrinth nach den operativen Eingriffen zu machen. Für die Vollkommenheit aller ausgefiihrten Exstirpationen ist der Beweis stets sowohl positiv durch die Untersuchung der exstirpirten Labyrinthbestandtheile, als auch negativ durch das Fehlen dieser Theile in den durch die Sectionen gewonnenen Präparaten erbracht worden.

Je nach der Länge der Zeit, die seit der Operation verflossen ist, finden sich die Verhältnisse im Labyrinth verändert. Die meisten der von mir auf secundäre Degeneration untersuchten Präparate stammen von Thieren, die innerhalb 6 Wochen post oper. getödtet sind.

An Stelle der nervösen Gebilde etc. findet sich dann die ganze Höhlung ausgefüllt von einem äusserst zarten, spinngewebartigen Bindegewebe, welches in feinen Strängen und Maschen die gegenseitigen Wandungen verbindet, zuweilen auch grössere Oeffnungen freilässt. Hin und wieder finden sich dazwischen noch scbwarze Schollen, Reste zerfallener Markscheiden, sowie andere Zerfallsproducte. Meist sind aber auch diese Gewebstrümmer bei dem bekannten schnellen Heilungsverlanfe bei Tauben bereits resorbirt.

Zahlreiche neu gebildete Capillaren durchziehen die dichten Gewebsstränge an den Resten der knöchernen Labyrinthwandungen. Die durch die Eingriffe abgelösten Muskelpartien haben an diesen vielfach vascularisirten Knochenresten neue Insertionsstellen gefunden.

Sind nur Theile des Labyrinthes herausgenommen, so verhalten sich die noch erhaltenen anseheinend vollkommen normal. 
Um die noch erhaltenen Partien bat sich eine Art neuer Labyrinthhöhle gebildet, ausgefüllt von Endolymphe, die Wandungen sind innen mit Epithelzellen ausgekleidet.

Bei den isolirten Schneckenexstirpationen hat sich das Lumen der knöchernen Schnecke gleichfalls mit schwarzen Massen, die von dichtem Bindegewebe durchzogen sind, ansgefuillt gefunden. Die sonst normal glatten, von dünnem Perioste ausgekleideten Wandungen sind buchtig, verzogen. Neugebildete Capillaren durchzieben das Ganze. In den oberen Partien, wahrscheinlich durch Resorption und Retraction einerseits, durch den lymphatischen Druck andererseits hervorgerufen, finden sich auch ein oder mehrere lu* mina vor. Ist die Columella noch drin geblieben, so ist ihre Platte tief in das Lumen der knöchernen Schnecke eingezogen und fixirt.

$\mathrm{Zu}$ erwähnen ist endlich noch, dass eine vollkommene Entfernung aller neryösen Gebilde nur schwer gelingt, desto leichter ist es aber, die nervösen Endapparate in toto herauszuziehen. Es liegt dies eben, wie es im Texte erwähnt ist, an dem Baue der Vogelschnecke. Um nun doch möglichst alle Ganglienzellen des Ganglion cochleare zu entfernen, bis der Stumpf des N. cochlearis sichtbar ist, wie ihn auch $\mathrm{E}$ wald gesehen hat, muss man wiederbolt mit einem entsprechenden Instrumente (krummes Häkchen oder kleines löffelartiges Instrument) eingehen. Man hat sich dabei nur davor zu hüten, dass nicht gleichzeitig das Vestibulum mit zerstört wird. Desbalb gelingt auch eine vollständige Ausräumung viel leichter bei Totalexstirpationen des häutigen Ohrlabyrinthes. Aber selbst wenn geringe Reste zurückgeblieben sind, erscheinen doch irgend welche Bedenken nicht gerechtfertigt. Denn wenn auch die nachfolgende Untersuchung noch einige Ganglienzellen nachweisen kann, so finden sich dieselben hochgradig alterirt, in dichtes nengebildetes Bindegewebe eingeschlossen, von Zerfallsproducten überbäuft ete. Von einer functionellen Bedeutung dieser Reste kann infolgedessen keine Rede sein.

\section{Erklärung der Abbildungen auf Tafel VI.}

1. Fignr 1 stellt ein Orientirungspräparat dar, um die Lage der Ursprungsganglien des N. acusticus zum Gehirn und zum Labyrinth zu 
zeigen. Das Präparat stammt von einem normalen Thiere. Nach der Entkalkung ist es in toto in Frontalschnitte zerlegt. Die Umrisse eines geeignetcn Schnittes - beide Hälften sind nicht ganz gleichmässig getroffen - sind bei 10 facher Lupenvergrösserung mit dem A b be'schen Zeichenapparate abgezeichnet. Nur die Details, welche zum Verständniss der Zeichnung erforderlich erschienen, sind bei stärkerer Vergrösserung eingetragen, von übrigen Angaben ist abgesehen worden.

Sehr deutlich zeigt der Schnitt die verschiedene Lage der Ganglien. Wichtig ist die Thatsache, dass das Ganglion cochleare $(g c)$ von aussen, etwa von der schematisch mit $o$ bezeichneten Stelle aus, entfernt werden kann, obne die das Gehirn schützende Knochenpartie $k$ aufzubrechen. Das Ganglion vestibulare $(g v)$ liegt dagegen intracraniell, ist also von aussen ohne besondere Eingriffe nicht zu erreichen.

A b k ïrzungen.

$$
\begin{array}{rlrl}
k l= & \text { knöchernes Labyrinth } & g c= & \text { Ganglion cochleare (roth) } \\
h l= & \text { häntiges Labyrinth } & p a c= & \text { papilla acustica cochleae } \\
& \text { (Kerne roth) }
\end{array}
$$

2. Figur 2 stellt ein Combinationspräparat nach den Methoden Weigert und Marchi-Algeri dar. Die Umrisse sind bei etwa 15 facher Vergrösserung (Zeiss) mit dem A b be'schen Zeichenapparat gezeichnet, die Details bei circa 4 mal stärkerer Vergrösserung eingetragen. Die normalen markhaltigen Fasern sind schwarz gezeichnet (Weigert), die degenerirenden Nervenfasern sind durch die characteristischen Zerfallsproducte (M a r c h iA 1 g e ri) deutlich.

Der Schnitt stellt einen Querschnitt der Med. obl. in der Höhe des Acusticuseintrittes dar bei einem doppelseitig labyrinthlosen Thiere mit hochgradigem Zerfalle der Nervenfasern. $a$ und $b$ sind die eintretenden Wurzelfasern, von denen $b$ im Verhältniss zu $a$ nur spärliche schwarze Schollen zeigt, während umgekehrt in $a$ (N. cochlearis) so gut wie keine normalen Nervenfasern zu bemerken sind. Es deutet dieser Unterschied anf die im Texte besprochene innige Durchkreuzung der Nervenfasern des $\mathrm{N}$. vestibularis und N. cochlearis kurz vor ihrem Eintritte ins verl. Mark. Die von der mehr vorn gelegenen Schnecke herkommenden zerfallenden Nervenfasern treten eben in der Hauptsache zu den weiter distalwärts gelegenen Kernen $c$ und $d$. Die Lage dieser Kerne $(c=$ "grosszelliger" Kern, $d=$ "Eckkern" nach $B$ randis) erscheint bei verschiedener Schnittführung verschieden, ebenso 
natürlich in verschiedener Schnitthöhe. Von diesen Kernen ziehen die Bogenzugfasern (Bgzg. = Striae acusticae) zur Raphe, um sich mit denen der andern Seite zu kreuzen (centrale Acusticusbahnen).

3. Figur 3 stellt den Eintritt des N. vestibularis (N. VIII e) ins Mark dar. Der Schnitt ist gleichfalls von einem doppelseitig labyrinthlosen Thiere gewonnen und unter denselben Bedingungen wie 2 gezeichnet.

Zwischen den starken normalen Nervenfasern finden sich constant auch degenerirende, über deren Bedeutung noch keine vollkommene Klarheit gewonnen werden konnte. Der Haupttheil der Fasern scheint zur Raphe hin zu strahlen. Weit weniger Fasern treten in Beziehung zu dem „kleinzelligen “ Kerne $f$, der auf einigen Präparaten von äusserst feinen schwarzen Körnchen bedeckt ist (zerfallende Collateralen?). Der Kern $d$ entspricht dem Kerne $d$ von Abbildung II, seine anscheinend veränderte Lage ist lediglich durch eine veränderte Schnittrichtung bedingt. Um diesen Kern herum sieht man anch eine verhältnissmässig reichere Anhäufung von schwarzen Schollen, was wohl auf seine intimere Beziehung zum N. cochlearis zurückzuführen ist.

Die übrigen Bezeichnungen ergeben sich von selbst.

(Aus dem physiologischen Institut der Universität Halle.)

\section{Ueber die specifische Energie des Hörnerven, die Wahrnehmung binauraler (diotischer) Schwebungen und die Beziehungen der Hörfunktion zur statischen Funktion des Ohrlabyrinths.}

Von

J. Bernstein.

Das grosse Interesse und die Wichtigkeit des in der vorangehenden Arbeit von Herrn Dr. M a t t e behandelten Gegenstandes sowie der Umstand, dass diese Arbeit unter meinen Augen ent- 